\title{
Barriers to gene flow play an important role in the speciation of two closely related Populus (Salicaceae) species
}

\author{
Yang Tian ${ }^{1}$, Shuyu Liu ${ }^{1}$, Pär Ingvarsson² ${ }^{2}$ Dandan Zhao ${ }^{1}$, Li Wang ${ }^{1}$, Baoerjiang \\ Abuduhamiti ${ }^{1}$, Zhiqiang $\mathrm{Wu}^{3}$, Jian-Guo ZHANG ${ }^{1}$, and Zhaoshan Wang ${ }^{1}$ \\ ${ }^{1}$ Chinese Academy of Forestry Research Institute of Forestry \\ ${ }^{2}$ Swedish University of Agricultural Sciences \\ ${ }^{3}$ Chinese Academy of Agricultural Sciences Agricultural Genomes Institute at Shenzhen
}

September 28, 2020

\begin{abstract}
Despite the growing number of recent studies on genome-wide divergence during speciation, the genetic basis and mechanisms of genomic divergence and speciation are still incompletely understood. In most species, natural selection plays a key role in genomic heterogeneous divergence. Additionally, barriers to gene flow, such as chromosomal rearrangements or gene incompatibilities, can also cause genome heterogeneity and speciation. Based on whole genome re-sequencing data from 27 Populus alba and $28 \mathrm{P}$. adenopoda individuals, we explored the reasons for the heterogeneous differentiation of genomes of these two closely related species. The results showed that the two species diverged $\sim 5-10$ million years ago (Mya), when the Qinghai-Tibet Plateau reached a certain height and the inland climate of the Asian continent became arid, which caused the two species begin to diverge and eventually led to speciation. In highly differentiated regions, neutrality tests (Tajima's D and Fay \& Wu's H) of these regions revealed no difference while the absolute divergence (dxy) were significantly higher than genome background, which indicates that barriers to gene flow rather than natural selection played a major role in maintaining genomic heterogeneous divergence and reproductive isolation, which is the most important condition for speciation. We further found that some genes related to reproduction may be involved in explaining the reproductive isolation of the two species.
\end{abstract}

\section{Introduction}

Understanding the origins of reproductive isolation is a major goal of modern speciation studies in plants because reproductive isolation is crucial for speciation (Cruickshank \& Hahn, 2014; Widmer, Lexer, \& Cozzolino, 2008). Postzygotic isolation may be caused by inferior niche adaptation, reduced attractiveness of hybrids or reduced fertility and/or viability of hybrids resulting from intrinsic incompatibilities (Eric, 2015; Levin, 1978; Lynch \& Force, 2000; MacNair \& Christie, 1983; H. Allen Orr \& Turelli, 2001; Qvarnström \& Bailey, 2008). Some degree of post-zygotic isolation often occurs in recently diverged species and are followed by the slow establishment of stronger prezygotic isolation (Eric, 2015; J. Wang, Street, Scofield, \& Ingvarsson, 2016; Widmer et al., 2008).

The origins of reproductive isolation include multiple evolutionary forces, including genetic drift, natural selection and mutations (Eric, 2015). Reproductive isolation is often considered to be an important by-product of genetic drift or differential adaptation when no gene flow is present (Noor, Grams, Bertucci, \& Reiland, 2001; Nosil, Egan, \& Funk, 2008). However, divergent selection in the face of gene flow is a common scenario that can have different effects across the genome of an organism (Nosil, Funk, \& Ortiz-barrientos, 2009). Positive and/or negative selection at individual loci can be reduced in one or both daughter populations, thereby increasing the degree of differentiation at these loci (Geraldes, Basset, Smith, \& Nachman, 2011; Rifkin, Castillo, Liao, \& Rausher, 2019). Selection on linked sites (due to genetic hitchhiking or background 
selection) can also lead to more differentiation in genomic regions with lower recombination rates (Nachman Michael \& Payseur Bret, 2012). In addition, phenomena such as chromosomal rearrangements or gene incompatibilities can also contribute to reproductive isolation (Baack, Melo, Rieseberg, \& Ortiz-Barrientos, 2015; Noor et al., 2001; Rieseberg, 2001; Schumer et al., 2014). Gene incompatibilities may lead to infertility or even mortality of hybrids between species and chromosomal rearrangements are known to play an important role in the origin of reproductive isolation because they sometimes disrupt meiosis in hybrids, resulting in inviability and/or infertility in hybrids (Hou, Friedrich, de Montigny, \& Schacherer, 2014; Lynch \& Force, 2000; Teresa Avelar, Perfeito, Gordo, \& Godinho Ferreira, 2013).

A common interpretation of the small number of strongly differentiated regions identified during genome scanning is that they represent loci related to reproductive isolation or ecological specialization and are that these loci are under strong selection to prevent introgression between species (Cruickshank \& Hahn, 2014; Payseur \& Rieseberg, 2016; Wu, 2001). Loci unrelated to reproductive isolation will experience homogenization from gene flow and will consequently exhibit low or no genetic differentiation. These differences in the action of natural selection is thought to explain a pattern of heterogeneous differentiation among sites in the genomes of closely related species (Cruickshank \& Hahn, 2014). There are many factors leading to heterogeneous genomic divergence (Nosil et al., 2009), including selection due to ecological causes (Funk et al., 2016) or genetic conflicts (Nosil et al., 2009; Presgraves, 2007), random effects of genetic drift (Funk et al., 2016), variable mutation rates (Cruickshank \& Hahn, 2014; Lynch \& Force, 2000) or the genomic distribution and effect size of selected genes and chromosome structure (Noor et al., 2001; Rieseberg, 2001; Teresa Avelar et al., 2013).

Therefore, it is important to analyse different driving factors to account for how heterogeneous genomic divergence is formed and what roles they play in the formation and maintenance of reproductive isolation and adaptive phenotypic differentiation (Jacobs, Hughes, Robinson, Adams, \& Elmer, 2018). In the absence of gene flow and natural selection between species that have recently diverged, the heterogeneous differentiation of the genome is simply resulting from stochastic variation in coalescence times (Barton, 2006). However, if selection is present, regions that experience strong natural selection will exhibit larger differences than those under weaker selection and will thus share less ancestral variation (Cruickshank \& Hahn, 2014). In the presence of gene flow, selection reduces the diversity of selected loci but also on sites linked to the selected loci thereby promoting genetic differentiation and resulting in an inflation of measures such as $F$ ST between species (Cruickshank \& Hahn, 2014; Jakobsson, Edge, \& Rosenberg, 2013; Walsh \& Blows, 2009). Natural selection reduces effective gene flow near selected sites; however, selection does not affect the redistribution of alleles in daughter species. Other regions exhibit low differentiation, either because they maintain a high level of ancestral polymorphism or because the homogenizing effects of gene flow, thereby leading to heterogenous genetic differentiation across the genome or an organism (Turner \& Hahn, 2010; J. Wang et al., 2016).

Barriers to gene flow, such as chromosomal rearrangements or gene incompatibilities, can also cause genome heterogeneity because they inhibit the redistribution of alleles between daughter species. Such barriers can lead to the formation of highly differentiated regions by countering the homogenizing effects of gene flow (Feder \& Nosil, 2009). At the same time, other areas of the genome are homogenized by gene flow, consequently showing lower levels of differentiation. This process results in a highly variable degree of genetic differentiation throughout the genome, also known as heterogeneous genomic differences (Nosil et al., 2009; Ortíz-Barrientos, Reiland, Hey, \& Noor, 2002; Wolf \& Ellegren, 2016). Chromosomal rearrangements are also known to reduce the fitness of heterozygotes via affecting meiosis, which may eventually result in reproductive isolation (Baack et al., 2015).

In summary, all three factors outlined above can explain heterogeneity in genomic differentiation and distinguishing between them and deciding which are the major factors in a particular scenario is a challenging task. Of course, these assumptions are not mutually exclusive and detailed information about the process of speciation, such as the time of speciation, the time of differentiation, and the demographic history of the species pair, are required to understand the causes of heterogeneous differentiation across the genome (Nosil et al., 2008; J. Wang et al., 2016). 
Populus alba and P. adenopoda are two important tree species in the section Populus with large ecological and economic value (Y. Wang et al., 2010). A phylogenetic tree constructed using a combination of cpDNA and nuclear DNA show that the two species are sister species (Z. Wang et al., 2014), indicating that they have diverged recently.P. alba, white poplar, is widely distributed and cultivated across the North African deserts, European flood plain forests and in central Asian regions having a strong continental climate and severe winter frosts (Dickmann \& Kuzovkina, 2014; Stölting et al., 2015). P. adenopoda is endemic to China, where it often occurs on sunny slopes or along riversides and is common in the southwest, where it shows good natural regeneration (L. Fan, Zheng, Milne, Zhang, \& Mao, 2018). Natural populations of P. alba often hybridise with populations of other, closely related species and hybridization with $P$. adenopoda, have produced a large number of natural $P$. tomentosa hybrids, which have poor fertility and produce almost no offspring (K. Li, Huang, \& Wang, 1997; Y. Wang et al., 2011). Therefore, while the two species have diverged recently they nevertheless show evidence for very strong postzygotic isolation. $P$. alba and $P$. adenopoda is thus a promising system for evaluating how different evolutionary forces contribute to the genomic patterns of divergence during speciation and how the accumulation of genetic differences leads to intrinsic barriers to reproduction (Palumbi \& R, 1994).

In this study, we used genome-wide re-sequencing data from the two species to infer their divergence time and estimate historical population dynamics. By examining population genomic statistics, such as genetic diversity and relative and absolute divergence, we expected to identify the major factors leading to heterogeneous genomic differentiation between the two species and to find outlier regions and genes that may be associated with adaptation to novel environments during the speciation process.

\section{Materials and Methods}

\section{Population samples, sequencing and mapping}

27 individuals of $P$. alba and 28 individuals of P. adenopoda were sampled (Figure 1a and Table S1). We extracted genomic DNA from leaves of each individual using a DNA extraction kit (Aidlab, Beijing, China). Paired-end (PE) reads were constructed according to the Illumina library preparation protocol and sequenced on an Illumina HiSeq 2000 platform (Illumina, San Diego, CA). The sequencing coverage target for all samples was $25 \times$. The data that support the findings of this study have been deposited into CNGB Sequence Archive (Guo et al., 2020) of CNGBdb with accession number CNP0001249 (http://db.cngb.org/cnsa/project/CNP0001249/reviewlink/). We obtained cleaned sequencing data from the sequencing company and then mapped to the P. trichocarpa reference genome (v3.0) (Tuskan et al., 2006) using bwa-0.7.15 with default settings. We then performed PCR duplication removal on BAM files using MarkDuplicates from the Picard toolkit. Only reads with the highest summed base quality were used for downstream analyses.

\section{Filtering sites}

We used several filtering steps to rule out errors that could be caused by paralogous or repeated DNA sequences before using the variant and genotype calls. First, we removed sites with a very low $(<13$ reads for each sample per species) or high (>100 reads for each sample per species) read coverage after examining the empirical distribution of reads. Second, since zero-mapped mass fractions were assigned to reads that could have been mapped to multiple genomic locations, we removed sites containing more than 20 such reads in all samples of each species. Third, we removed sites that overlapped with known repetitive elements identified using RepeatMasker (N. Chen, 2004; Tarailograovac \& Chen, 2009). After these filtering steps, $48.6 \%$ of the sites in the genome were used for downstream analysis.

\section{SNP and genotype calling}

Although ANGSD has been shown to be superior to the genotype calling methods in SAMtools and GATK (Depristo et al., 2011; H. Li et al., 2009), we used two complementary methods for SNP and genotype calling: i) Direct estimation of the genotype without calling in ANGSD v0.602 (Korneliussen et al., 2014). We considered only reads with a minimum mapping quality of 30 and bases with a minimum quality score of 
20. For all the filtered sites in these two species, we defined the allele that was identical to that found in the P. trichocarpa reference genome as the ancestral allele. Based on the SAMtools genotype likelihood model for all sites, we used -doSaf to calculate the site allele frequency likelihood and then used -realSFS for maximum likelihood estimation of the expanded SFS using the expectation maximization (EM) algorithm (Su et al., 2011). Then, we calculated several population genetic statistics based on the global SFS, such as Fay \& Wu's H; the proportions of shared, private and fixed polymorphisms; and population structure parameters. ii) Multi-sample SNP and genotype calls were performed using HaplotypeCaller and GenotypeGVCFs in GATK by performing a series of filtering steps on the output VCF file. We also ran a number of filtering steps to decrease false positives from SNP and genotype calls. We deleted SNPs in regions that did not pass all previous screening conditions and SNPs with more than two alleles in both species. Furthermore, if a genotype's quality (GQ) was less than 10, it was designated as a missing genotype, and then the SNPs with more than two missing genotypes in each species were filtered out.

\section{Population structure}

The NGSadmix program (Skotte et al., 2013) was used to infer population genetic structure by selecting only sites with less than $10 \%$ missing data. Based on the output from ANGSD, it was possible to infer individual admixture proportions and estimate the frequency of different ancestral populations based on genotype likelihoods. We utilized the SAMtools model (H. Li et al., 2009) in ANGSD to determine genotype likelihood and then generated a Beagle file of the genome subset using a likelihood ratio test (Su et al., 2011). The number of genetic clusters $\mathrm{K}$ was predetermined and varied from 2 to 4 and the maximum iteration of the EM algorithm was set to 10,000 .

Similarly, the sample allele frequency likelihoods generated in ANGSD were used to perform a PCA to visualize inter-individual genetic relationships included possible sequencing errors and all uncertainties in the genotype calls (Fumagalli, Vieira, Linderoth, \& Nielsen, 2014). The expected covariance matrix of each individual in the two species was calculated according to the genotypic posterior probability of all the filtered sites.

\section{Demographic history}

We used fastSimcoal2 (ver 2.6.0.3-14.10.17) (Excoffier et al., 2013) to infer the demographic histories associated with the speciation of $P$. alba and P. adenopoda based on the coalescent simulation method. A two-dimensional joint SFS (2D-SFS) was constructed using the posterior probability from ngsTools for the frequency of the sample allele. We used 100,000 joint simulations to estimate the expected 2D-SFS and $\log$ probabilities for a set of demographic parameters in each model. The global maximum likelihood estimates for each model were obtained by 50 independent runs. We compared the models based on the maximum likelihood of 50 independent runs using the Akaike information criterion (AIC) and Akaike's evidence weights. The model with the largest Akaike's weight value was chosen as the best model. We assumed that the mutation rate of poplar was $2.5 \times 10^{-9}$ per year and that the generation time was 15 years when converting estimates to years and individual units. The CI of the best model was obtained from 100 parametric bootstrap samples, with 50 independent runs for each sample. Then we used the PSMC method (H. Li \& Durbin, 2011) to estimate historical changes in the population size of the two species. We used beagle.08Jun17.d8b.jar to phase and impute all of the sites within each species before analysis. The scaled time and population size were converted to actual time and size using a 15-year generation time and $2.5 \times 10^{-9}$ mutations per nucleotide per year.

\section{Genome-wide patterns of differentiation}

We divided the genome into 39,406 non-overlapping 10kbp windows for studying the pattern of genomic differentiation between the species. After all filtering steps detailed above, a window was used for downstream analyses if it included at least 1,000 bases. We also removed windows where the number of variable sites were less than 10.F ST was calculated using the software VCFtools (v0.1.13) (Danecek et al., 2011) to estimate the degree of genetic differentiation between species at each site. 


\section{transformed $\boldsymbol{F}_{\mathrm{ST}}$ values to find outlier regions}

VCFtools (v0.1.13) (Danecek et al., 2011) was used to calculate the relative measure of divergence $F$ sT and then we utilized the Origin 8 software to perform Z-transformation $F$ sT. We chose to set the thresholds at $\mathrm{Z}\left(F_{\mathrm{ST}}\right)>1.96$ and $\mathrm{Z}\left(F_{\mathrm{ST}}\right)<-1.96$ as high and low-differentiated regions, 415 and 983 outlier windows were classified as highly and lowly differentiated regions, respectively. We compared two outlier windows (highly differentiated and lowly differentiated regions) with the rest of the genome by analysing additional population genetic statistics of the two species. First, we calculated the values of $\vartheta \pi$, Tajima's D and Fay \& Wu's $\mathrm{H}$ according to the sample allele frequency likelihood of the non-overlapping 10-Kbp windows in ANGSD. Furthermore, we estimated and compared LD levels and recombination rates based on population size and used VCFtools (Danecek et al., 2011) to calculate the correlation coefficients $\left(r^{2}\right)$ between SNPs with a distance greater than $1 \mathrm{Kbp}$ to evaluate the level of LD in each 10-Kbp window. Finally, we utilized the ngsStat (Fumagalli et al., 2014) program to calculate several other genetic differentiation parameters: (1) the proportion of fixed differences caused by the fixation of derived alleles in $P$. alba and $P$. adenopoda, using $P$. trichocarpa as an outgroup; (2) the proportion of shared polymorphisms among species at all segregating sites; (3) the absolute measure of divergence $\left(\mathrm{d}_{\mathrm{xy}}\right)$ based on the posterior probability of the sample allele frequency for each locus and then averaged for each 10-Kbp window; and (4) RND, calculated by dividing the $\mathrm{d}_{\mathrm{xy}}$ between $P$. alba and $P$. adenopoda by the $\mathrm{d}_{\mathrm{xy}}$ between the two poplars and $P$. trichocarpa . For this process, we used one-sided Wilcoxon ranked-sum tests to detect significant differences between values in outlier windows and the genome-wide mean of all population genetic statistics.

\section{GO enrichment}

To determine if any functional gene classes were overexpressed in the outlier regions with barriers to gene flow, we performed functional enrichment analysis of GO terms using Fisher's exact test (https://www.omicshare.com/tools/Home/Soft/gogsea). This analysis excluded GO groups with fewer than two genes and further corrected the p-value from Fisher's exact test using the Benjamini-Hochberg false discovery rate (Benjamini \& Hochberg, 1995). GO terms with a modified $P$-value $<0.05$ were considered significantly enriched.

\section{Results}

A total of $27 P$. alba and $28 P$. adenopoda individuals were selected for whole-genome re-sequencing. The $P$. trichocarpagenome (v3.0) was used as the reference genome because of its high-quality assembly and annotation as well as the high genomic synteny between members of sections Populus ( $P$. alba and $P$. adenopoda ) and Tacamahaca (P. trichocarpa) (Liu, Wang, \& Zeng, 2019; Pakull, Groppe, Meyer, Markussen, \& Fladung, 2009; Tuskan et al., 2006). After removing reads containing adaptors, trimming and filtering, the sequenced reads from the two species were mapped to the P. trichocarpa reference genome (v3.0) (Tuskan et al., 2006) with a moderately high mapping rate ( $84.3 \%$ on average, Table S1). The mean coverage of reads that were uniquely mapped per site was 23.9 and 24.8 in $P$. alba and $P$. adenopoda, respectively (Table S1). In this study, we used two complementary bioinformatics methods. First, the genetic statistics of populations that depended on the inferred site-frequency spectrum (SFS) were estimated directly from the genotype likelihoods rather than by calling the genotype (Nielsen, Korneliussen, Albrechtsen, Li, \& Wang, 2012), in ANGSD (Korneliussen, Albrechtsen, \& Nielsen, 2014). Second, HaplotypeCaller in GATK (Danecek et al., 2011) was used to call single nucleotide polymorphisms (SNPs) and genotypes for estimations that required accurate genotype calls. VCFtools (Danecek et al., 2011) was used to perform a series of filtering steps on the VCF files prior to data analysis. A total of 22,525,740 and 21,217,984 high-quality SNPs were obtained from the $27 \mathrm{P}$. alba and $28 \mathrm{P}$. adenopoda samples, respectively, after filtering.

\section{Population structure and Demographic histories}

NGSadmix (Skotte, Korneliussen, \& Albrechtsen, 2013) was used for quantifying admixture proportions for the two species. The program infers individual ancestry based on genotype likelihoods and examines the genetic relationships between individuals. The analyses were run with the number of genetic clusters (K) varying from 2 to 4 . When $\mathrm{K}=2$ (Figure $1 \mathrm{~b}$ ), all samples were divided into two species-specific popula- 
tions. When $\mathrm{K}=3$ (Figure $1 \mathrm{~b}$ ), the results were no longer meaningful. This result was verified by principal component analysis (PCA) that show a clear separation of the two species and little evidence for further subdivision within species (Figure 1c).

We usedfastsimcoal2.6(Excoffier, Dupanloup, Huerta-Sánchez, Sousa, \& Foll, 2013), which is a continuoustime coalescence simulator assuming arbitrarily complex evolutionary scenarios (Excoffier \& Foll, 2011), to infer the evolutionary history of $P$. alba and P. adenopoda . 25 different divergence models were assessed (Figure S1; Table S2). All models began with the subdivision of an ancestral population into two derived groups and thereafter differed in the following ways: (I) divergence time, (II) the occurrence and duration of gene flow following population divergence, and (III) effective population size before and after differentiation. The most appropriate model was an isolation-migration-isolation model, where the separation of the two species involved three phases (Figure 2a). The exact parameter estimates were provided for this model in Table 1, including the divergence time, levels of gene flow and effective population sizes together with their associated $95 \%$ confidence intervals (CIs). Under the best fitting model, $P$. alba and $P$. adenopoda diverged from an ancestral population approximately 9.54 million years ago (Mya) (bootstrap range [BR]: 5-10 Mya), and this differentiation initially occurred in allopatry as there was no evidence for gene flow during the early stages of differentiation. From 1.15 Mya to 0.34 Mya there was evidence for secondary contact and weak asymmetric gene flow between the two incipient species, after which the two species returned to an allopatric state without gene flow. The population size of the two isolated species showed stepwise changes, with $P$. alba experiencing a population decline during in the final stages. The migration rate of $P$. alba to $P$. adenopoda per generation was $1.28 \times 10^{-6}\left(7.49 \times 10^{-7}-2.33 \times 10^{-6}\right)$, and that from $P$. adenopoda to $P$. alba was $9.69 \times 10^{-6}\left(7.45 \times 10^{-6}-1.40 \times 10^{-5}\right)$. This result was not surprising because the geographical distance between the two species was large and their distributions were very scattered. The estimates of contemporary effective population size $(N e)$ of $P$. alba $\left(N_{e-P . ~ a l b a}\right)$ and P. adenopoda $\left(N_{e-P . \text { adenopoda }}\right)$ were 19,551 (BR:18624.523509.0) and 31,333 (BR:30851.5-34287.8), respectively, both of which were larger than the common ancestor which was 11,506 (BR:3170.7-80404.5).

We also used the pairwise sequentially Markovian coalescent (PSMC) model (H. Li \& Durbin, 2011) to reconstruct historical effective population size changes in the two species with a focus on diploid consensus sequences. Estimates of the effective population sizes of $P$. albaand $P$. adenopoda based on the PSMC model at the beginning of population divergence approximately 10 Mya were similar to those of their ancestral population obtained with fastsimcoal2.6. The two species experienced similar degrees of population expansions following divergence, possibly indicating successful adaptation to new niches (Figure 2b). The population size of $P$. alba then began to decline but rebounded with a second population expansion from 1.15-0.34 Mya. In contrast, the population of $P$. adenopoda has undergone considerable and continuous decline (Figure $2 \mathrm{~b}$ ).

\section{Genome-wide patterns of differentiation and identification of outlier regions}

We used $F_{\text {ST }}$, a relative measure of divergence (B Charlesworth, 1998), to explore inter-specific genomic differentiation patterns in non-overlapping 10-kilobase (Kbp) windows (Figure 3). As shown in Figure 3, genetic differentiation varied widely across the genome with an average $F$ st value of 0.542 and many windows showed substantially higher genetic differentiation between species. We identified 415 and 983 outlier windows which showed significantly $(P<0.05)$ high and low $F$ ST values, respectively (Figure 4a). By examining the genomic distribution of outlier windows, we determined that both highly and lowly differentiated regions were randomly distributed throughout the genome (Figure 3).

Since $F$ sTis based on a comparison of intra- and inter-population diversity, any reduction in the former will increase $F$ ST $($ Nachman Michael \& Payseur Bret, 2012). We used three other methods to quantify and compare inter-specific genetic differentiation between the two classes of outlier windows and the rest of the genome. The first was $\mathrm{d}_{\mathrm{xy}}$, an absolute measure of divergence, which was the average number of pairwise differences between the two populations (Cruickshank \& Hahn, 2014). The second was relative node depth (RND), which was $\mathrm{d}_{\mathrm{xy}}$ divided by $\mathrm{d}_{\mathrm{xy}}$ relative an outgroup species, in this case $P . t$ richocarpa . This ratio corrected for possible variation in the mutation rate among genomic regions. The third was the proportion of ancestral polymorphisms shared by the two species. We found that $\mathrm{d}_{\mathrm{xy}}$ and RND were significantly higher 
(Figure 4b; Table S3) and that the proportion of shared ancestral polymorphisms was significantly lower in the highly differentiated regions of the two species compared to the genomic background (Figure 4b; Table S3). These regions also contained a greater proportion of fixed differences, caused by the fixation of derived alleles in both species relative to the genome-wide average (Figure 4c; Table S3). Both species showed positive Tajima's D values and negative Fay \& Wu's H values on average. The values of Tajima's D and Fay \& Wu's H test showed no significant difference when comparing highly differentiated regions to the genomic background in the two species. Linkage disequilibrium (LD) in highly differentiated regions also showed no significant difference to the genomic background. However, when we divided the genome into three subsets based on $\mathrm{d}_{\mathrm{xy}}$ values (high part, top 1000; medium and low part, bottom 1000), the $\mathrm{d}_{\mathrm{xy}}$ values in the high and low parts showed significantly lower and higher value than the background in both the two species, respectively (Figure 4c; Table S3).

In contrast to the pattern found in highly differentiated regions, polymorphism in the lowly differentiated regions was higher. The value of Tajima's D test and Fay \& Wu's H test were significantly higher for lowly differentiated regions than background regions. Compared with the genomic background, the proportion of polymorphisms shared between species was higher in lowly differentiated regions and the proportion of fixed differences was extremely low (Figure 4b, c; Table S3). We also investigated the correlation between relative and absolute divergence between $P$. alba and $P$. adenopoda and found a strong positive correlation between them (Spearman's $p=0.623, P$-value $<0.001$ ).

A GO functional analysis of regions with high or $\operatorname{low} F$ st values was performed using the $P$. trichocarpa genome annotation. We assessed whether specific GO terms were significantly overexpressed using gene ontology (GO) assignments of these candidate genes. 293 and 599 genes were identified in the outlier windows showing high differentiation or low genetic differentiation, respectively (Table S4 and S5). We identified 41 GO categories showing significant enrichment, with the most prominent group (7 terms) associated with the term "reproduction" $(P<0.05)$ (Table S6). Genes related to reproduction and adaptation in highly differentiated regions between $P$. alba and P. adenopodaare listed on Tables S7 and S8.

\section{Discussion}

\section{Demography}

We took advantage of genome-wide patterns of genetic diversity and differentiation to explore the evolutionary history of the two closely related species $P$. alba and $P$. adenopoda to study how the genomic heterogeneity in genetic diversity and differentiation is affected by various evolutionary forces.

Demographic modelling showed that $P$. alba and P. adenopo da diverged approximately 9.5 Mya (5 Mya-10 Mya), during the late Miocene. The climate of Asia has been strongly linked to the surface uplift of the Tibetan Plateau (J. Li et al., 2014; J. X. Li et al., 2018; W. Li \& Chetelat, 2014; Zhang, Fengquan, \& Jianmin, 2000; Zhisheng, Kutzbach, Prell, \& Porter, 2001). The Asian winter monsoon has appeared in some form since about 13.1 Mya (M. Fan, Song, Dettman, Fang, \& Xu, 2006). Rising vapour from the Indian Ocean cools, condenses and precipitates before it crosses the Tibetan Plateau, which results in drier air advancing to the northern plateau (Fang et al., 2019; Favre et al., 2015; Ge, 2006; J. X. Li et al., 2018). The combination of mountains and monsoons makes the climate in north-western China drier and the aridity reached its maximum approximately 9.6-8.0 Mya (C. Chen et al., 2019; M. Fan, Dettman, Song, Fang, \& Garzione, 2007; Mertz-Kraus, Brachert, Jochum, Reuter, \& Stoll, 2009; Zhisheng et al., 2001). From 5.3 Mya the climate has gradually became drier and/or cooler (M. Fan et al., 2007). Furthermore, the uplift of the Pamir Plateau and the central plateau of Anatolia, coupled with the collision between the Pamir Plateau and the Tianshan Mountains, further blocked the transport of water vapour from the winter westerly belt to the east 5 Mya (J. X. Li et al., 2018; Meijers et al., 2018; Shen et al., 2018; Thompson, Burbank, Li, Chen, \& Bookhagen, 2015). Approximately 2-3 Mya, the climate was still very dry and cold because north-western China received only the water vapour in the westerly belt at that time (Caves et al., 2015; X. Li et al., 2017; Zhuang, Brandon, Pagani, \& Krishnan, 2014). Approximately 1.80-1.10 Mya, the paleotemperature was approximately $7.5-13{ }^{\circ} \mathrm{C}$ (Meijers et al., 2018), which was not sufficient to cause contact between $P$. alba 
and $P$. adenopoda. In our demographic modelling this is evident from the lack of gene flow that occurred during the early stages of divergence of the two species (from 9.54 Mya to 1.15 Mya).

Our modelling detects a small amount of asymmetric gene flow between the two species $\sim 1.15-0.34$ Mya. This time period was close to the appearance of the Mindel-Riss Interglacial (Jing \& Liu, 1999; Wright, 1926), which also included several cold and warm periods (Cheng, 1996; Lindner, 1981; Peiyuan, 1989). During this period, the paleotemperature increased significantly and the annual average temperature reached 28 ${ }^{\circ} \mathrm{C}$ (Cheng, 1996; Peiyuan, 1989). A form of subtropical forest or forest grassland environment was present during this time period and the climate was much warmer and wetter than in modern times. Based on the results of the PSMC simulation, the population of $P$. alba experienced exponential size changes at this stage. From $~ 0.34$ Mya to the present, gene flow between P. alba and P. adenopoda has been interrupted again and this period corresponds to the Riss Glacial (Jing \& Liu, 1999; Kukla, 2005).

\section{Heterogeneous differentiation across the genome}

Relative divergence measures, such as $F_{\mathrm{ST}}$, are based on a comparison of within-population diversity to between-population diversity (B Charlesworth, 1998; Cruickshank \& Hahn, 2014). Absolute divergence is usually measured as $d_{x y}$, the average number of pairwise differences between alleles sampled from two populations (Nachman Michael \& Payseur Bret, 2012), and variation in $\mathrm{d}_{\mathrm{xy}}$ (T. Ma et al., 2018) can be caused by variation in levels of ancestral polymorphism or substitution rates (Cruickshank \& Hahn, 2014). A more comprehensive understanding of heterogeneity in genomic differentiation requires the use of both relative (influenced by within-population variation) and absolute (not influenced by within-population variation) measures of sequence divergence (Cruickshank \& Hahn, 2014; Wolf \& Ellegren, 2016). Any type of selection that reduces levels of linked neutral diversity will lead to increased estimates of relative divergence (such as F $\mathrm{ST}$ ) (B Charlesworth, 1998; Brian Charlesworth, Nordborg, \& Charlesworth, 1997; Cruickshank \& Hahn, 2014). However, the increased $F$ ST seen in such regions does not always indicate a reduction in actual gene flow. In other words, there is a redistribution of alleles in these regions. Therefore, the value of $\mathrm{d}_{\mathrm{xy}}$ in these regions does not necessarily increase. In contrast, if barriers to gene flow are the main driving force of heterogeneous differentiation across the genome, both absolute and relative divergence measures should be high because the redistribution of alleles in these regions is prevented while the remainder of the genome is homogenized by gene flow.

Most studies have implicated natural selection as the primary cause for genomic heterogeneous differentiation and even for speciation (Cruickshank \& Hahn, 2014; Nosil, Vines, \& Funk, 2005). Selection affects population differentiation by reducing polymorphism levels at sites under selection (Cruickshank \& Hahn, 2014). The size of differentiated regions is related to the intensity of selection and the stage of species divergence and the highly differentiated region may represent genes associated with reproductive isolation (Wu, 2001). However, barriers to gene flow, such as chromosomal rearrangements and genetic incompatibilities can also lead to heterogeneous differentiation of genomes (Faria \& Navarro, 2010; Navarro, Betran, Barbadilla, \& Ruiz, 1997; Navarro \& Ruiz, 1997; Rieseberg, 2001). Barriers to gene flow inhibits allele exchange in such regions and in linked surrounding regions eventually lead to elevated genetic differentiation. If such highly differentiated regions contain genes associated with reproductive isolation, speciation may eventually occur (Cruickshank \& Hahn, 2014; $\mathrm{Wu}, 2001)$. Our results found that absolute divergence $\left(\mathrm{d}_{\mathrm{xy}}\right)$ were strongly positively correlated with relative divergence $(F \mathrm{ST})$ and this finding implies that barriers to gene flow have played a major role in generating the heterogeneous differentiation between $P$. alba and $P$. adenopoda, although natural selection cannot be thoroughly ruled out.

In this study, we Z-transformed the $F$ sT distributions to identify regions showing high, medium and low genetic differentiation across the genome. We evaluated multiple population genetic parameters to understand the mechanisms generating the heterogeneous genomic differentiation seen between the two species. Overall the values of Tajima's D in the two species were positive and Fay \& Wu's H were negative, suggesting that recent population contractions have taken place (Fay \& Wu, 2000; Holliday, Yuen, Ritland, \& Aitken, 2010; Tajima, 1989), consistent with the PSMC results (Figure 2b). Both of the two neutrality tests (Tajima's D and Fay \& Wu's H) showed no difference for the highly differentiated regions in either of the two species, 
suggesting that selection hasn't been the major factor in the maintenance of highly differentiated regions.

The extremely high correlation between relative and absolute divergence highlights the significant effects of barriers to gene flow in generating the heterogeneous differentiation landscape between the two species. Furthermore, when we divided the whole genome into three parts by $d_{x y}$ values we found that the high part showed significantly lower correlation coefficients $\left(r^{2}\right)$ value than the genomic background, further supporting the conclusion that barriers to gene flow play an important role in shaping the genome-wide divergence of the two species (Ellegren et al., 2012; Geraldes et al., 2011). That may because when the foreign DNA fragments were introduced by introgression, the observed value of correlation coefficients $\left(r^{2}\right)$ increased in related regions, but not for the regions of barriers to gene flow because the foreign DNA fragment cannot be integrated into the genome. Compared to the genomic background, highly differentiated regions also contained an excess of derived fixed differences and a smaller proportion of shared polymorphisms. In addition, the nucleotide diversity $(\vartheta \pi)$ in highly differentiated regions was significantly greater than that of the genomic background, consistent with views outlined above.

\section{Reproductive isolation and speciation}

Reproductive isolation is a consequence of prezygotic and postzygotic barriers as well as their potentially complex interactions (Rieseberg \& Willis, 2007; Widmer et al., 2008). Prezygotic isolation, due to reduced probabilities for meeting (spatial or temporal isolation), mating (sexual isolation) or successful fertilization (homogametic sperm preferences or sperm-egg incompatibilities), has been found to be a greater contributor to the total isolation between species than postzygotic isolation (Qvarnstrom \& Bailey, 2009).

Postzygotic isolation can be caused by intrinsic and extrinsic factors, leading to phenomena such as hybrid inviability and sterility and ecological and behavioural sterility, respectively (Widmer et al., 2008). $P$. tomentosa, one of the most widely distributed species in China, is a natural hybrid between $P$. alba and $P$. adenopoda (K. Li et al., 1997; Z. Wang et al., 2014) and while P. tomentosa has strong adaptability is has poor fertility and high abortion rates (D. Wang, Wang, Kang, \& Zhang, 2019). A recent study using more than 200 individuals of $P$. tomentosafound no progeny resulting from backcrossing or selfing. Furthermore, some studies have found that $P$. tomentosa exhibits unequal numbers of univalents and a decreased degree of synapsis during meiosis (Kang, 2001). A larger percentage of univalents occurs at diakinesis and metaphase I and at anaphase I and telophase I lagging chromosomes are frequently observed (Kang, Zhu, \& Zhang, 1999). This finding is consistent with the conclusion of this study that heterogeneous genomic differentiation caused by barriers to gene flow is an important force in speciation. All of the above results confirmed that $P$. alba and $P$. adenopoda are subject to postzygotic isolation and imply that barriers to gene flow have played an important role in speciation of the two species because they can establish and maintain reproductive isolation.

There are several reasons for postzygotic barriers to gene flow, such as chromosomal rearrangements or gene incompatibilities (King \& Templeton, 1993; Noor et al., 2001). Structural changes in the genome, including deletions, insertions, duplications, inversions and translocations, alter the genome organization of individuals and may also contribute to reproductive isolation (Wolf \& Ellegren, 2016). Genomic incompatibilities might result from chromosomal rearrangements, which lead to mis-segregation during meiosis in hybrids, or from epistatic interactions that act as loss-of-function alleles in hybrid backgrounds (Lynch \& Force, 2000). These factors are often accompanied by a decrease in hybrid inviability, sterility and/or even mortality (Barbash, Siino, Tarone, \& Roote, 2003; H. Allen Orr, 1989; H. A. Orr \& Coyne, 1989; Schumer et al., 2014). To distinguish to what extent chromosomal rearrangements and gene incompatibilities contribute to postzygotic isolation, high-quality genomes for the two species must be assembled in the future.

To further categorise genes located in outlier regions, we performed gene ontology (GO) analysis in order to identify gene ontology terms that were enriched in these gene sets. We identified 41 categories that showing significant enrichment and the most prominent group ( 7 terms) was associated with the word "reproduction". The seven genes belonging to this category include $C D C$ 2,CUL 1, emb 2746, AFO ,HK 2, TFL 2 and EDA 8 , several of which are known to affect cell division, pollen rejection pathways, embryogenesis, flowers/spike into 
seedlings and reproductive habits, plant germ cell development during meiosis, transition from photoperiod regulation to reproductive growth and the early development of plant embryos and pollen tubes (Table S9). These findings support the hypothesis that highly differentiated regions contain an excess of reproductionrelated genes. The gene ontology analysis also identified three genes involved in seed germination and a gene for drought resistance. $R C A R 3$ and $G C R 1$ regulated seed germination and early seedling development while $C P K 23$ is involved in drought- and salt stress-induced calcium signaling cascades (Colucci, Apone, Alyeshmerni, Chalmers, \& Chrispeels, 2002; Lim, Luan, \& Lee, 2014; S.-Y. Ma \& Wu, 2007). These genes may therefore be signatures of differential adaptation in the two species, P. albaand P. adenopo da .

\section{Acknowledgments}

We thank Dong Wang, Aiguo Duan, Wenhao Shao, Sirong Yi, Zhonghui Shi, Bin Zhang for some sample collection. We thank Prof. Jing Wang in Sichuan University, Associate Professor. Fumin Zhang, Dr. Zhe Cai and Chunyan Jing in the State Key Laboratory of Systematic and Evolutionary Botany, Institute of Botany, Chinese Academy of Sciences, Beijing, China, for their valuable suggestion on data analysis. This work was supported by China National GeneBank (CNGB).

\section{Data Accessibility}

Whole genome re-sequencing data of Populus species are available at http://db.cngb.org/cnsa/.

\section{Author Contributions}

Zhaoshan Wang and Jianguo Zhang designed the experiments. Yang Tian, Shuyu Liu, Dandan Zhaoa and Li Wang performed the experiments. Baoerjiang Abuduhamiti was responsible for sampling. Pär K. Ingvarsson and Zhiqiang Wu put forward guidance. Zhaoshan Wang and Yang Tian analysed data and contributed to writing the manuscript.

\section{References}

Baack, E., Melo, M. C., Rieseberg, L. H., \& Ortiz-Barrientos, D. (2015). The origins of reproductive isolation in plants. New Phytologist, 207 (4), 968-984. doi:10.1111/nph.13424

Barbash, D. A., Siino, D. F., Tarone, A. M., \& Roote, J. (2003). A rapidly evolving MYB-related protein causes species isolation in Drosophila. Proceedings of the National Academy of Sciences, 100 (9), 5302. doi:10.1073/pnas.0836927100

Barton, N. H. (2006). Evolutionary Biology: How Did the Human Species Form? Current Biology, 16 (16), R647-R650. doi:https://doi.org/10.1016/j.cub.2006.07.032

Benjamini, Y., \& Hochberg, Y. (1995). Controlling the False Discovery Rate: A Practical and Powerful Approach to Multiple Testing. Journal of the Royal Statistical Society: Series B (Methodological), 57 (1), 289-300. doi:10.1111/j.2517-6161.1995.tb02031.x

Caves, J. K., Winnick, M. J., Graham, S. A., Sjostrom, D. J., Mulch, A., \& Chamberlain, C. P. (2015). Role of the westerlies in Central Asia climate over the Cenozoic. Earth and Planetary Science Letters, 428 , 33-43. doi:https://doi.org/10.1016/j.epsl.2015.07.023

Charlesworth, B. (1998). Measures of divergence between populations and the effect of forces that reduce variability. Molecular Biology and Evolution, 15 (5), 538-543. doi:10.1093/oxfordjournals.molbev.a025953

Charlesworth, B., Nordborg, M., \& Charlesworth, D. (1997). The effects of local selection, balanced polymorphism and background selection on equilibrium patterns of genetic diversity in subdivided populations. Genetical Research, 70 (2), 155-174. doi:10.1017/S0016672397002954

Chen, C., Bai, Y., Fang, X., Guo, H., Meng, Q., Zhang, W., . . Murodov, A. (2019). A Late Miocene Terrestrial Temperature History for the Northeastern Tibetan Plateau's Period of Tectonic Expansion. Geophysical Research Letters, 46 (14), 8375-8386. doi:10.1029/2019GL082805 
Chen, N. (2004). Using RepeatMasker to Identify Repetitive Elements in Genomic Sequences. Current protocols in human genetics, 25 (1).

Cheng, Z. (1996). Comparative Study on the Sedimentary Environment of the Fourth Period in Lushan, Huangshan and Tianmu Mountain Areas. Geographic science, 016 (1), 37-45.

Colucci, G., Apone, F., Alyeshmerni, N., Chalmers, D., \& Chrispeels, M. J. (2002). GCR1, the putative Arabidopsis G protein-coupled receptor gene is cell cycle-regulated, and its overexpression abolishes seed dormancy and shortens time to flowering. Proceedings of the National Academy of Sciences, 99 (7), 47364741. doi:10.1073/pnas.072087699

Cruickshank, T. E., \& Hahn, M. W. (2014). Reanalysis suggests that genomic islands of speciation are due to reduced diversity, not reduced gene flow. Molecular Ecology, 23 (13), 3133-3157.

Danecek, P., Auton, A., Abecasis, G., Albers, C. A., Banks, E., DePristo, M. A., . . Sherry, S. T. (2011). The variant call format and VCFtools. Bioinformatics, 27 (15), 2156-2158.

Depristo, M. A., Banks, E., Poplin, R., Garimella, K. V., Maguire, J., Hartl, C., . . Hanna, M. (2011). A framework for variation discovery and genotyping using next-generation DNA sequencing data. Nature Genetics, 43 (5), 491-498.

Dickmann, D., \& Kuzovkina, Y. (2014). Poplars and Willows in the World. In (pp. 8-91).

Ellegren, H., Smeds, L., Burri, R., Olason, P. I., Backström, N., Kawakami, T., . . Wolf, J. B. W. (2012). The genomic landscape of species divergence in Ficedula flycatchers. Nature, 491 , 756. doi:10.1038/nature11584 https://www.nature.com/articles/nature11584\#supplementary-information

Eric, B. (2015). The origins of reproductive isolation in plants. The New phytologist, 4 (207).

Excoffier, L., Dupanloup, I., Huerta-Sánchez, E., Sousa, V. C., \& Foll, M. (2013). Robust Demographic Inference from Genomic and SNP Data.PLOS Genetics, 9 (10), e1003905. doi:10.1371/journal.pgen.1003905

Excoffier, L., \& Foll, M. (2011). fastsimcoal: a continuous-time coalescent simulator of genomic diversity under arbitrarily complex evolutionary scenarios. Bioinformatics, 27 (9), 1332-1334. doi:10.1093/bioinformatics/btr124

Fan, L., Zheng, H., Milne, R. I., Zhang, L., \& Mao, K. (2018). Strong population bottleneck and repeated demographic expansions of Populus adenopoda (Salicaceae) in subtropical China. Annals of Botany, 121 (4), 665-679. doi:10.1093/aob/mcx198

Fan, M., Dettman, D. L., Song, C., Fang, X., \& Garzione, C. N. (2007). Climatic variation in the Linxia basin, NE Tibetan Plateau, from 13.1 to 4.3 Ma: The stable isotope record. Palaeogeography, Palaeoclimatology, Palaeoecology, 247 (3), 313-328. doi:https://doi.org/10.1016/j.palaeo.2006.11.001

Fan, M., Song, C., Dettman, D. L., Fang, X., \& Xu, X. (2006). Intensification of the Asian winter monsoon after 7.4 Ma: Grain-size evidence from the Linxia Basin, northeastern Tibetan Plateau, 13.1 Ma to 4.3 Ma. Earth and Planetary Science Letters, 248 (1), 186-197. doi:https://doi.org/10.1016/j.epsl.2006.05.025

Fang, X., Fang, Y., Zan, J., Zhang, W., Song, C., Appel, E., . . . Zhang, T. (2019). Cenozoic magnetostratigraphy of the Xining Basin, NE Tibetan Plateau, and its constraints on paleontological, sedimentological and tectonomorphological evolution. Earth-Science Reviews, 190 , 460-485. doi:https://doi.org/10.1016/j.earscirev.2019.01.021

Faria, R., \& Navarro, A. (2010). Chromosomal speciation revisited: rearranging theory with pieces of evidence. Trends in Ecology and Evolution, 25 (11), 660-669.

Favre, A., Päckert, M., Pauls, S. U., Jähnig, S. C., Uhl, D., Michalak, I., \& Muellner-Riehl, A. N. (2015). The role of the uplift of the Qinghai-Tibetan Plateau for the evolution of Tibetan biotas.Biological Reviews, 90 (1), 236-253. doi:10.1111/brv.12107 
Fay, J. C., \& Wu, C. (2000). Hitchhiking Under Positive Darwinian Selection. Genetics, 155 (3), 1405-1413.

Feder, J. L., \& Nosil, P. (2009). Chromosomal inversions and species differences: when are genes affecting adaptive divergence and reproductive isolation expected to reside within inversions? Evolution, 63 (12), 30613075. doi:10.1111/j.1558-5646.2009.00786.x

Fumagalli, M., Vieira, F. G., Linderoth, T., \& Nielsen, R. (2014). ngsTools: methods for population genetics analyses from next-generation sequencing data. Bioinformatics, 30 (10), 1486-1487. doi:10.1093/bioinformatics/btu041

Funk, W. C., Lovich, R. E., Hohenlohe, P. A., Hofman, C. A., Morrison, S. A., Sillett, T. S., . . Andelt, W. F. (2016). Adaptive divergence despite strong genetic drift: genomic analysis of the evolutionary mechanisms causing genetic differentiation in the island fox (Urocyon littoralis). Molecular Ecology, 25 (10), 2176-2194. doi: $10.1111 /$ mec. 13605

Ge, X. (2006). Multi-stage uplifts of the Qinghai-Tibet plateau and their environmental effects. Earth Sci. Frontiers, 13 (6), 118-130.

Geraldes, A., Basset, P., Smith, K. L., \& Nachman, M. W. (2011). Higher differentiation among subspecies of the house mouse (Mus musculus) in genomic regions with low recombination. Molecular Ecology, 20 (22), 4722-4736. doi:10.1111/j.1365-294X.2011.05285.x

Guo, X., Chen, F., Gao, F., Li, L., Liu, K., You, L., . . Xu, X. (2020). CNSA: a data repository for archiving omics data.bioRxiv , 2020.2004.2007.030833. doi:10.1101/2020.04.07.030833

Holliday, J. A., Yuen, M., Ritland, K., \& Aitken, S. N. (2010). Postglacial history of a widespread conifer produces inverse clines in selective neutrality tests. Molecular Ecology, 19 (18), 3857-3864.

Hou, J., Friedrich, A., de Montigny, J., \& Schacherer, J. (2014). Chromosomal Rearrangements as a Major Mechanism in the Onset of Reproductive Isolation in Saccharomyces cerevisiae. Current Biology, 24 (10), 1153-1159. doi:https://doi.org/10.1016/j.cub.2014.03.063

Jacobs, A., Hughes, M. R., Robinson, P. C., Adams, C. E., \& Elmer, K. R. (2018). The Genetic Architecture Underlying the Evolution of a Rare Piscivorous Life History Form in Brown Trout after Secondary Contact and Strong Introgression. Genes, 9 (6), 280.

Jakobsson, M., Edge, M. D., \& Rosenberg, N. A. (2013). The Relationship Between FST and the Frequency of the Most Frequent Allele.Genetics, 193 (2), 515. doi:10.1534/genetics.112.144758

Jing, C., \& Liu, H. (1999). On the glacial and interglacial stages in Quaternary of China. J. Cheng Du Univ. Tech, $26,97-100$.

Kang, X. (2001). Study on mechanism of pollen abortion in chinese white poplar (Populus tomentosa carr.). Scientia Silvae Sinicae.

Kang, X., Zhu, Z., \& Zhang, Z. (1999). Cytogenetic studies on the origin of Chinese white poplar. Journal of Beijing Forestry University, 21 (6), 6-10.

King, M., \& Templeton, A. R. (1993). Species Evolution: The Role of Chromosome Change. 44 (4), 191-202.

Korneliussen, T. S., Albrechtsen, A., \& Nielsen, R. (2014). ANGSD: Analysis of Next Generation Sequencing Data. BMC Bioinformatics, 15 (1), 356. doi:10.1186/s12859-014-0356-4

Kukla, G. (2005). Saalian supercycle, Mindel/Riss interglacial and Milankovitch's dating. Quaternary Science Reviews, 24 (14), 1573-1583. doi:https://doi.org/10.1016/j.quascirev.2004.08.023

Levin, D. A. (1978). The Origin of Isolating Mechanisms in Flowering Plants. In M. K. Hecht, W. C. Steere, \& B. Wallace (Eds.),Evolutionary Biology (pp. 185-317). Boston, MA: Springer US. 
Li, H., \& Durbin, R. (2011). Inference of human population history from individual whole-genome sequences. Nature, 475 (7357), 493-496. doi:10.1038/nature10231

Li, H., Handsaker, B., Wysoker, A., Fennell, T., Ruan, J., Homer, N., . . G Genome Project Data Processing, S. (2009). The Sequence Alignment/Map format and SAMtools. Bioinformatics, 25 (16), 2078-2079. doi:10.1093/bioinformatics/btp352

Li, J., Fang, X., Song, C., Pan, B., Ma, Y., \& Yan, M. (2014). Late Miocene-Quaternary rapid stepwise uplift of the NE Tibetan Plateau and its effects on climatic and environmental changes. Quaternary Research, 81 (3), 400-423. doi:10.1016/j.yqres.2014.01.002

Li, J. X., Yue, L. P., Roberts, A. P., Hirt, A. M., Pan, F., Guo, L., . . Liu, Q. S. (2018). Global cooling and enhanced Eocene Asian mid-latitude interior aridity. Nature Communications, 9 (1), 3026. doi:10.1038/s41467018-05415-x

Li, K., Huang, M., \& Wang, M. (1997). Study on origin of Populus tomemtosa Carr. Acta Phytotaxonomica Sinica, 35 (1), 24-31.

Li, W., \& Chetelat, R. T. (2014). The Role of a Pollen-Expressed Cullin1 Protein in Gametophytic SelfIncompatibility in Solanum.Genetics, 196 (2), 439-442.

Li, X., Hao, Q., Wei, M., Andreev, A. A., Wang, J., Tian, Y., . . Shi, W. (2017). Phased uplift of the northeastern Tibetan Plateau inferred from a pollen record from Yinchuan Basin, northwestern China.Scientific Reports, 7 (1), 18023. doi:10.1038/s41598-017-16915-z

Lim, C. W., Luan, S., \& Lee, S. C. (2014). A Prominent Role for RCAR3-Mediated ABA Signaling in Response to Pseudomonas syringae pv. tomato DC3000 Infection in Arabidopsis. Plant and Cell Physiology, 55 (10), 1691-1703. doi:10.1093/pcp/pcu100

Lindner, L. (1981). Organogenic deposits of the Mazovian Interglacial (Mindel II /Riss I) in the middle Vistula basin, compared to coeval European localities. Acta Geologica Polonica, 31 , 111-126.

Liu, Y.-J., Wang, X.-R., \& Zeng, Q.-Y. (2019). De novo assembly of white poplar genome and genetic diversity of white poplar population in Irtysh River basin in China. Science China Life Sciences, 62 (5), 609-618.

Lynch, M., \& Force, A. G. (2000). The Origin of Interspecific Genomic Incompatibility via Gene Duplication. The American Naturalist, 156 (6), 590-605. doi:10.1086/316992

Ma, S.-Y., \& Wu, W.-H. (2007). AtCPK23 functions in Arabidopsis responses to drought and salt stresses. Plant Molecular Biology, 65 (4), 511-518. doi:10.1007/s11103-007-9187-2

Ma, T., Wang, K., Hu, Q., Xi, Z., Wan, D., Wang, Q., . . Liu, J. (2018). Ancient polymorphisms and divergence hitchhiking contribute to genomic islands of divergence within a poplar species complex.Proceedings of the National Academy of Sciences, 115 (2), E236. doi:10.1073/pnas.1713288114

MacNair, M. R., \& Christie, P. (1983). Reproductive isolation as a pleiotropic effect of copper tolerance in Mimulus guttatus? Heredity, 50 (3), 295-302. doi:10.1038/hdy.1983.31

Meijers, M. J. M., Brocard, G. Y., Cosca, M. A., Lüdecke, T., Teyssier, C., Whitney, D. L., \& Mulch, A. (2018). Rapid late Miocene surface uplift of the Central Anatolian Plateau margin. Earth and Planetary Science Letters, 497 , 29-41. doi:https://doi.org/10.1016/j.epsl.2018.05.040

Mertz-Kraus, R., Brachert, T. C., Jochum, K. P., Reuter, M., \& Stoll, B. (2009). LA-ICP-MS analyses on coral growth increments reveal heavy winter rain in the Eastern Mediterranean at 9 Ma. Palaeogeography, Palaeoclimatology, Palaeoecology, 273 (1), 25-40. doi:https://doi.org/10.1016/j.palaeo.2008.11.015

Nachman Michael, W., \& Payseur Bret, A. (2012). Recombination rate variation and speciation: theoretical predictions and empirical results from rabbits and mice. Philosophical Transactions of the Royal Society B: 
Biological Sciences, 367 (1587), 409-421. doi:10.1098/rstb.2011.0249

Navarro, A., Betran, E., Barbadilla, A., \& Ruiz, A. (1997). Recombination and gene flux caused by gene conversion and crossing over in inversion heterokaryotypes. Genetics, 146 (2), 695-709.

Navarro, A., \& Ruiz, A. (1997). On the Fertility Effects of Pericentric Inversions. Genetics, 147 (2), 931.

Nielsen, R., Korneliussen, T., Albrechtsen, A., Li, Y., \& Wang, J. (2012). SNP Calling, Genotype Calling, and Sample Allele Frequency Estimation from New-Generation Sequencing Data. PLOS ONE, 7 , e37558. doi:10.1371/journal.pone.0037558

Noor, M. A. F., Grams, K. L., Bertucci, L. A., \& Reiland, J. (2001). Chromosomal inversions and the reproductive isolation of species.Proceedings of the National Academy of Sciences, 98 (21), 12084. doi: $10.1073 /$ pnas. 221274498

Nosil, P., Egan, S. P., \& Funk, D. J. (2008). Heterogeneous genomic differentiation between walking-stick ecotypes: "isolation by adaptation" and multiple roles for divergent selection.Evolution, 62 (2), 316-336. doi:10.1111/j.1558-5646.2007.00299.x

Nosil, P., Funk, D. j., \& Ortiz-barrientos, D. (2009). Divergent selection and heterogeneous genomic divergence. Molecular Ecology, 18 (3), 375-402. doi:10.1111/j.1365-294X.2008.03946.x

Nosil, P., Vines, T. H., \& Funk, D. J. (2005). Reproductive isolation caused by natural selection against immigrants from divergent habitatats. Evolution, 59 (4), 705-719. doi:10.1111/j.0014-3820.2005.tb01747.x

Orr, H. A. (1989). Genetics of sterility in hybrids between two subspecies of drosophila. Evolution, 43 (1), 180-189. doi:10.1111/j.1558-5646.1989.tb04216.x

Orr, H. A., \& Coyne, J. A. (1989). The Genetics of Postzygotic Isolation in the Drosophila Virilis Group. Genetics, 121 (3), 527-537.

Orr, H. A., \& Turelli, M. (2001). The evolution of postzygotic isolation: accumulating dobzhansky-muller incompatibilities.Evolution, 55 (6), 1085-1094. doi:10.1111/j.0014-3820.2001.tb00628.x

Ortíz-Barrientos, D., Reiland, J., Hey, J., \& Noor, M. A. F. (2002). Recombination and the divergence of hybridizing species. In W. J. Etges \& M. A. F. Noor (Eds.), Genetics of Mate Choice: From Sexual Selection to Sexual Isolation (pp. 167-178). Dordrecht: Springer Netherlands.

Pakull, B., Groppe, K., Meyer, M., Markussen, T., \& Fladung, M. (2009). Genetic linkage mapping in aspen (Populus tremula L. and Populus tremuloides Michx.). Tree Genetics 83 Genomes, 5 (3), 505-515. doi:10.1007/s11295-009-0204-2

Palumbi, \& R, S. (1994). Genetic Divergence, Reproductive Isolation, and Marine Speciation. Annu Rev Ecol Syst, 25 (1), 547-572.

Payseur, B. A., \& Rieseberg, L. H. (2016). A genomic perspective on hybridization and speciation. Molecular Ecology, 25 (11), 2337-2360. doi:10.1111/mec.13557

Peiyuan, H. (1989). Discussion on Paleotemperature Change from the Ratio of High-priced Iron to Lowpriced Iron in the Quaternary Strata of Lushan Mountain. Published by the Institute of Geomechanics, Chinese Academy of Geological Sciences (2), 61-70.

Presgraves, D. C. (2007). Does genetic conflict drive rapid molecular evolution of nuclear transport genes in Drosophila? BioEssays, 29 (4), 386-391. doi:10.1002/bies.20555

Qvarnstrom, A., \& Bailey, R. I. (2009). Speciation through evolution of sex-linked genes. Heredity, 102 (1), $4-15$.

Qvarnström, A., \& Bailey, R. I. (2008). Speciation through evolution of sex-linked genes. Heredity, 102 , 4. doi:10.1038/hdy.2008.93 
Rieseberg, L. H. (2001). Chromosomal rearrangements and speciation. Trends in Ecology and Evolution, 16 (7), 351-358.

Rieseberg, L. H., \& Willis, J. H. (2007). Plant speciation.Science, 317 (5840), 910-914.

Rifkin, J. L., Castillo, A. S., Liao, I. T., \& Rausher, M. D. (2019). Gene flow, divergent selection and resistance to introgression in two species of morning glories (Ipomoea). Molecular Ecology, 28 (7), 17091729. doi: $10.1111 / \mathrm{mec} .14945$

Schumer, M., Cui, R., Powell, D. L., Dresner, R., Rosenthal, G. G., \& Andolfatto, P. (2014). High-resolution mapping reveals hundreds of genetic incompatibilities in hybridizing fish species. eLife, 3 . Retrieved from http://europepmc.org/abstract/MED/24898754

http://europepmc.org/articles/PMC4080447?pdf=render

http://europepmc.org/articles/PMC4080447

https://www.ncbi.nlm.nih.gov/pmc/articles/pmid/24898754/?tool=EBI

https://www.ncbi.nlm.nih.gov/pmc/articles/pmid/24898754/pdf/?tool=EBI

https://doi.org/10.7554/eLife.02535 doi:10.7554/eLife.02535

Shen, X., Wan, S., Colin, C., Tada, R., Shi, X., Pei, W., . . Li, A. (2018). Increased seasonality and aridity drove the C4 plant expansion in Central Asia since the Miocene-Pliocene boundary. Earth and Planetary Science Letters, 502 , 74-83. doi:https://doi.org/10.1016/j.epsl.2018.08.056

Skotte, L., Korneliussen, T. S., \& Albrechtsen, A. (2013). Estimating Individual Admixture Proportions from Next Generation Sequencing Data.Genetics, 195 (3), 693. doi:10.1534/genetics.113.154138

Stölting, K. N., Paris, M., Meier, C., Heinze, B., Castiglione, S., Bartha, D., \& Lexer, C. (2015). Genomewide patterns of differentiation and spatially varying selection between postglacial recolonization lineages of Populus alba (Salicaceae), a widespread forest tree.New Phytologist, 207 (3), 723-734. doi:10.1111/nph.13392

Su, Y. K., Lohmueller, K. E., Albrechtsen, A., Li, Y., Korneliussen, T., Geng, T., . . Witte, D. (2011). Estimation of allele frequency and association mapping using next-generation sequencing data. BMC Bioinformatics, 12 .

Tajima, F. (1989). Statistical method for testing the neutral mutation hypothesis by DNA polymorphism. Genetics, 123 (3), 585.

Tarailograovac, M., \& Chen, N. (2009). UNIT 4.10 Using RepeatMasker to Identify Repetitive Elements in Genomic Sequences. Current protocols in human genetics .

Teresa Avelar, A., Perfeito, L., Gordo, I., \& Godinho Ferreira, M. (2013). Genome architecture is a selectable trait that can be maintained by antagonistic pleiotropy. Nature Communications, 4 , 2235. doi:10.1038/ncomms3235

https://www.nature.com/articles/ncomms3235\#supplementary-information

Thompson, J. A., Burbank, D. W., Li, T., Chen, J., \& Bookhagen, B. (2015). Late Miocene northward propagation of the northeast Pamir thrust system, northwest China. Tectonics, 34 (3), 510-534. doi:10.1002/2014TC003690

Turner, T. L., \& Hahn, M. W. (2010). Genomic islands of speciation or genomic islands and speciation? Molecular Ecology, 19 (5), 848-850. doi:10.1111/j.1365-294X.2010.04532.x

Tuskan, G. A., DiFazio, S., Jansson, S., Bohlmann, J., Grigoriev, I., Hellsten, U., . . . Rokhsar, D. (2006). The genome of black cottonwood,Populus trichocarpa (Torr. \& Gray). Science, 313 (5793), 1596. doi:10.1126/science.1128691 
Walsh, B., \& Blows, M. W. (2009). Abundant Genetic Variation + Strong Selection = Multivariate Genetic Constraints: A Geometric View of Adaptation. Annual Review of Ecology, Evolution, and Systematics, 40 (1), 41-59. doi:10.1146/annurev.ecolsys.110308.120232

Wang, D., Wang, Z., Kang, X., \& Zhang, J. (2019). Genetic analysis of admixture and hybrid patterns of Populus hopeiensis and P. tomentosa.Scientific Reports, 9 (1), 4821. doi:10.1038/s41598-019-41320-Z

Wang, J., Street, N. R., Scofield, D. G., \& Ingvarsson, P. K. (2016). Variation in Linked Selection and Recombination Drive Genomic Divergence during Allopatric Speciation of European and American Aspens. Molecular Biology and Evolution, 33 (7), 1754-1767. doi:10.1093/molbev/msw051

Wang, Y., Sun, X., Tan, B., Zhang, B., Xu, L.-a., Huang, M., \& Wang, M. (2010). A genetic linkage map of Populus adenopoda Maxim. $\times$ P. alba L. hybrid based on SSR and SRAP markers. Euphytica, 173 (2), 193-205. doi:10.1007/s10681-009-0085-3

Wang, Y., Zhang, B., Sun, X., Tan, B., Xu, L.-a., Huang, M., \& Wang, M. (2011). Comparative genome mapping among Populus adenopoda, P. alba, P. deltoides, P. euramericana and P. trichocarpa . Genes 86 Genetic Systems, 86 (4), 257-268. doi:10.1266/ggs.86.257

Wang, Z., Du, S., Dayanandan, S., Wang, D., Zeng, Y., \& Zhang, J. (2014). Phylogeny Reconstruction and Hybrid Analysis of Populus (Salicaceae) Based on Nucleotide Sequences of Multiple Single-Copy Nuclear Genes and Plastid Fragments. PLOS ONE, 9 (8), e103645. doi:10.1371/journal.pone.0103645

Widmer, A., Lexer, C., \& Cozzolino, S. (2008). Evolution of reproductive isolation in plants. Heredity, 102 , 31. doi:10.1038/hdy.2008.69

Wolf, J. B. W., \& Ellegren, H. (2016). Making sense of genomic islands of differentiation in light of speciation. Nature Reviews Genetics, 18 , 87. doi:10.1038/nrg.2016.133

https://www.nature.com/articles/nrg.2016.133\#supplementary-information

Wright, W. B. (1926). The German Outwash Terraces and the Astronomical Theory of the Ice Age. Nature, 117 (2934), 113-114. doi:10.1038/117113a0

Wu, C.-I. (2001). The genic view of the process of speciation. Journal of Evolutionary Biology, 14 (6), 851-865. doi:10.1046/j.1420-9101.2001.00335.x

Zhang, D., Fengquan, L., \& Jianmin, B. (2000). Eco-environmental effects of the Qinghai-Tibet Plateau uplift during the Quaternary in China. Environmental Geology, 39 (12), 1352-1358. doi:10.1007/s002540000174

Zhisheng, A., Kutzbach, J. E., Prell, W. L., \& Porter, S. C. (2001). Evolution of Asian monsoons and phased uplift of the Himalaya-Tibetan plateau since Late Miocene times. Nature, 411 (6833), 62-66. doi: $10.1038 / 35075035$

Zhuang, G., Brandon, M. T., Pagani, M., \& Krishnan, S. (2014). Leaf wax stable isotopes from Northern Tibetan Plateau: Implications for uplift and climate since 15 Ma. Earth and Planetary Science Letters, 390 , 186-198. doi:https://doi.org/10.1016/j.epsl.2014.01.003

\section{F igures and Tables}

Figure 1. Sample collection and population structure analysis of $27 \mathrm{P}$. alba and $28 \mathrm{P}$. adenopoda. (a) 28 P. adenopoda individuals (red) were collected in Anhui, Chongqing, Hunan and Tianmushan of China and 27 P. alba individuals (blue) were collected from the Altay area, Xinjiang (a) (b) Population genetic structure in the samples based on an analysis using NGSadmix based on genotype likelihoods. The $\mathrm{y}$-axis represents the number of clusters, and the x-axis shows the name of each individual. (c) Results from a principal component analysis (PCA) on the genetic covariance matrix for all individuals of $P$. alba (blue circles) and $P$. adenopoda(red circles). 
Figure 2. Demographic history of $\boldsymbol{P}$. alba and $\boldsymbol{P}$. adenopoda. (a) The most appropriate model inferred by fastsimcoal2.6 . The ancestral population is colored gray while $P$. alba and the P. adenopoda are colored red and blue, respectively. The widths represents the relative effective population sizes $(N$ e). Double-headed arrows represents the per generation gene flow between $P$. alba and $P$. adenopoda. All inferred demographic parameters are presented in Table 1. (b) Pairwise Sequentially Markovian coalescent (PSMC) estimates of the effective population size $(N$ e) changes for $P$. alba (bold blue line) and $P$. adenopoda (bold red line) based on the inference from four phased haplotypes in each species, and performed 100 bootstrap replicates for four individuals (light-colored line respectively). Time scale on the $\mathrm{x}$-axis is calculated assuming a neutral mutation rate per generation $(\mu)=2.5 \times 10-9$ and generation time $(\mathrm{g})=15$ years.

Figure 3. Genome-wide divergence. Chromosomal distribution of pairwise genetic divergence $(F$ ST $)$ between $P$. alba and $P$. adenopoda in 10-kb sliding windows. Windows with significantly high or low genetic differentiation are shown in red and green, respectively.

Figure 4. Identifying candidate outlier windows affected by factors of barriers to gene flow. (a) Distribution of genetic differentiation $(F \mathrm{ST})$ between $P$. alba and $P$. adenopoda. The dotted line represents the threshold based on Z-scores to determine windows with high (red) $(P>0.05)$ or low (green) $(P<-0.05)$ genetic differentiation. (b) $\mathrm{d}_{\mathrm{xy}}$ (absolute measure of divergence), relative node depth (RND) and interspecific shared polymorphisms were compared between the genomic background and regions with significant high and low genetic differentiation. (c) Comparison of population genetic statistics between regions with significantly high (red) or low (green) genetic differentiation and the genomic background (blue) in $P$. alba and $P$. adenopoda . Statistics include Tajima's D, Fay \&Wu's H, the proportion of fixed differences caused by derived alleles, nucleotide diversity $(\vartheta \pi)$, and values of $d_{x y}$ and $F$ st calculated using the ${ }^{2}$, respectively. Asterisks indicate significant differences between outliers and background genomic regions based on Mann-Whitney $\mathrm{U}$ tests $\left(* P\right.$-value $<0.05 ;{ }^{* *} P$-value $<1 \mathrm{e}-4 ; * * * P$-value $\left.<2.2 \mathrm{e}-16\right)$.

Table 1. Demographic parameter estimates for the best-supported model in Figure 2a.

\begin{tabular}{llll}
\hline Point estimation 95\% CI & Point estimation 95\% CI & Point estimation 95\% $\mathrm{CI}^{\mathrm{a}}$ & Point estimation 95\% $\mathrm{CI}^{\mathrm{a}}$ \\
\hline Parameters & & Lower bound & Upper bound \\
$N_{\text {e-ANC }}$ & 11506 & 3170.675 & 80404.47 \\
$N_{\text {e-POP0 }}$ & 19551 & 18624.45 & 23508.97 \\
$N_{\text {e-POP1 }}$ & 30851.53 & 34287.77 \\
MIG01 & 31333 & $7.49 \times 10^{-7}$ & $2.33 \times 10^{-6}$ \\
MIG10 & $1.28 \times 10^{-6}$ & $7.45 \times 10^{-6}$ & $1.40 \times 10^{-5}$ \\
TDIV01 & $9.59 \times 10^{-6}$ & 21118.95 & 27847.42 \\
TDIV02 & 22786 & 50890.35 & 98329.9 \\
TDIV03 & 76468 & 336770.3 & 668025.3 \\
\hline
\end{tabular}

The parameters are defined in Figure 2a. $N_{\text {e-POP0 }}, N_{\text {e-POP1 }}$ and $N_{\text {e-ANCrepresent the effective population }}$ size of $P$. alba , P. adenopoda and their ancestral population respectively. MIG01 represents the migration rate of each generation from $P$. alba to $P$. adenopoda, and that from $P$. adenopoda to $P$. albais MIG10. TDIV01, TDIV02 and TDIV03 represents the estimated divergence time between the two species obtained from fastsimcoal2. 6 .

aParameter bootstrap estimation obtained by performing parameter estimation from 100 simulated data sets based on the total maximum composite likelihood estimates displayed in the point estimation column, per likelihood is estimated from 100,000 simulations. 
(a)
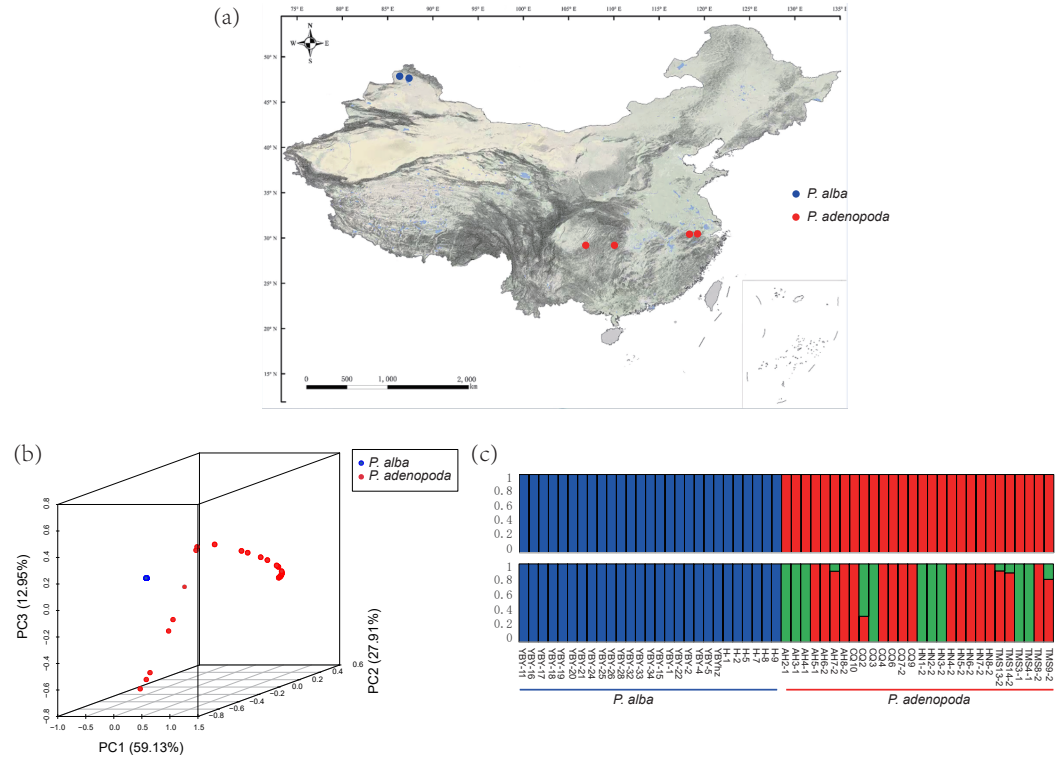
(a)

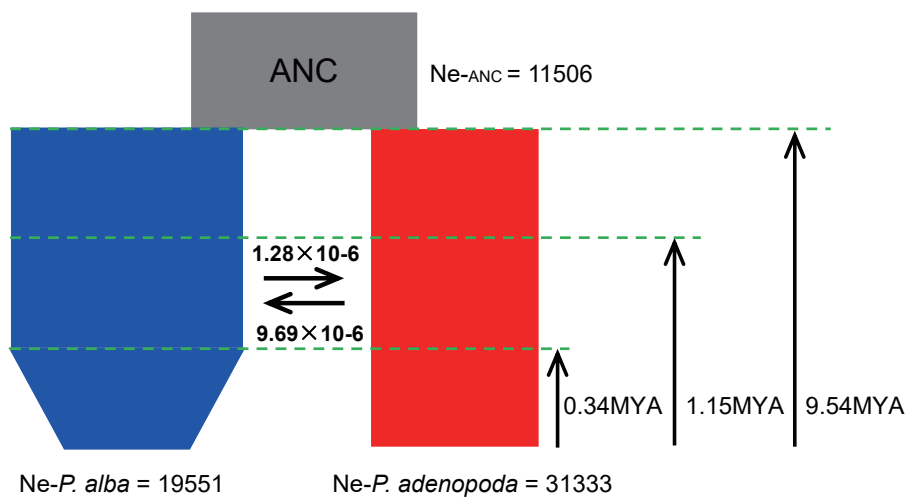

(b)

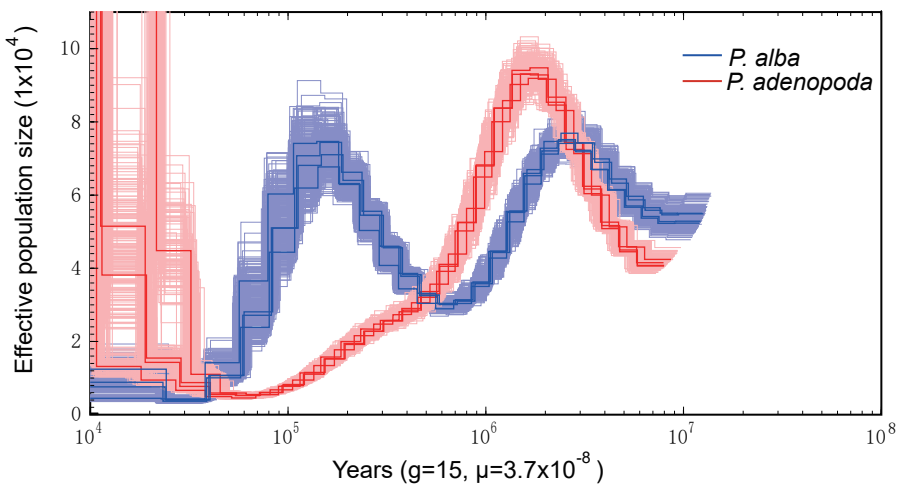




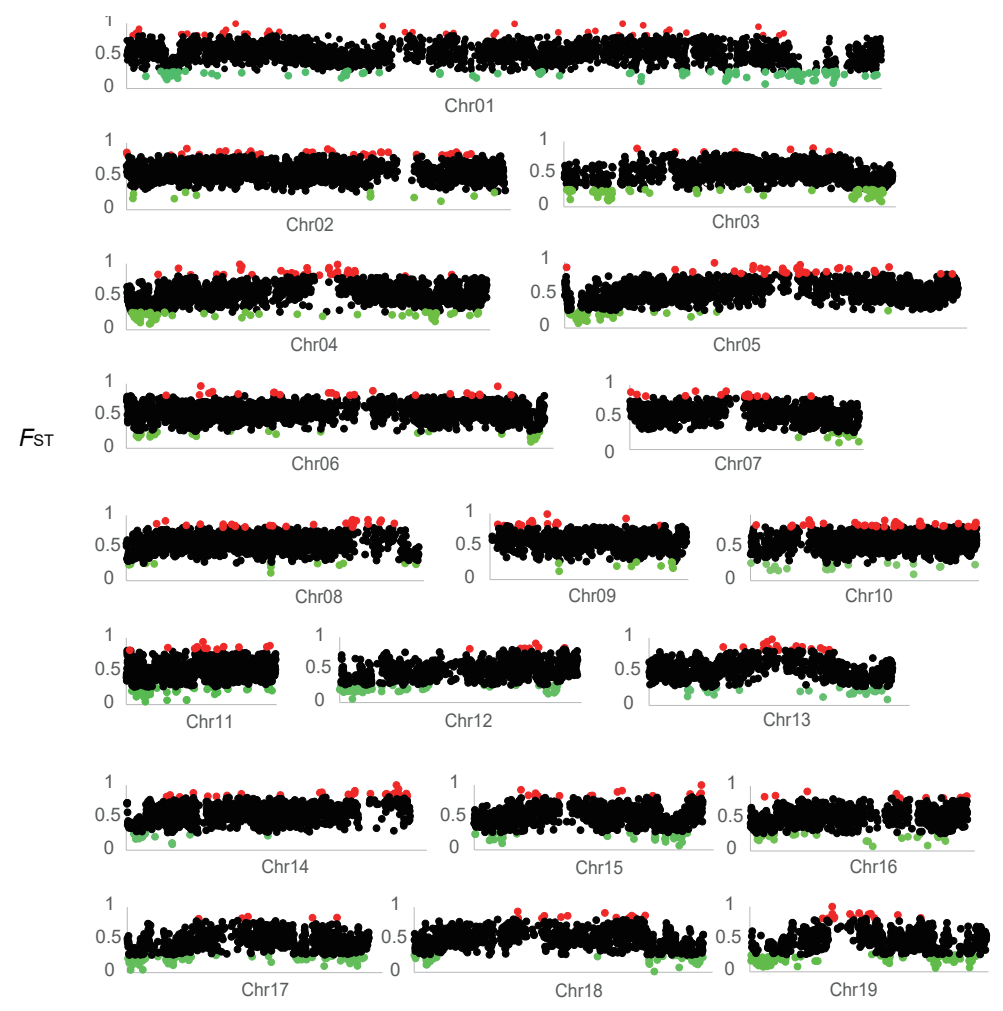


(a)

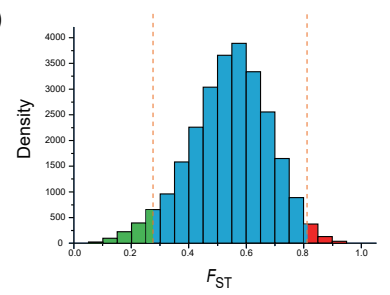

(b)

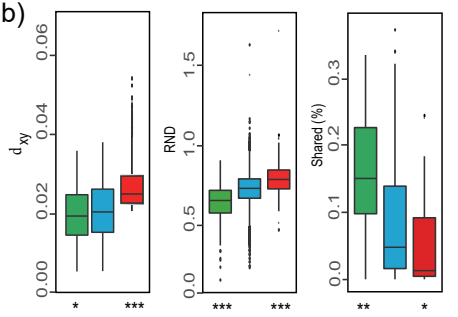

(c)
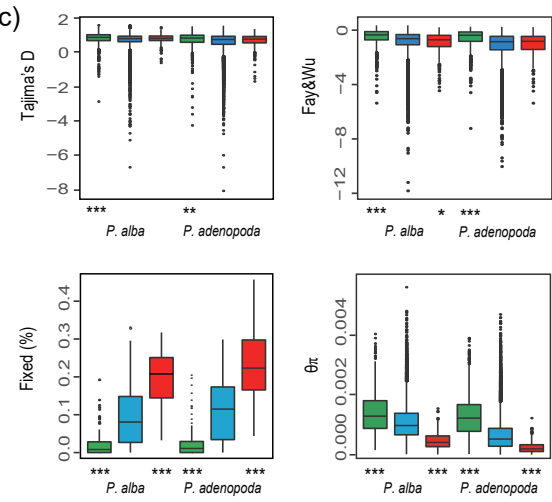

$\underset{\text { P. alba }}{\substack{* * \text { P. adenopoda } \\ 0}}$

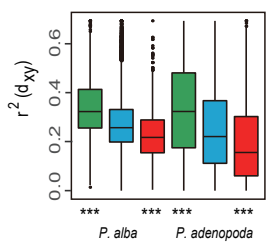



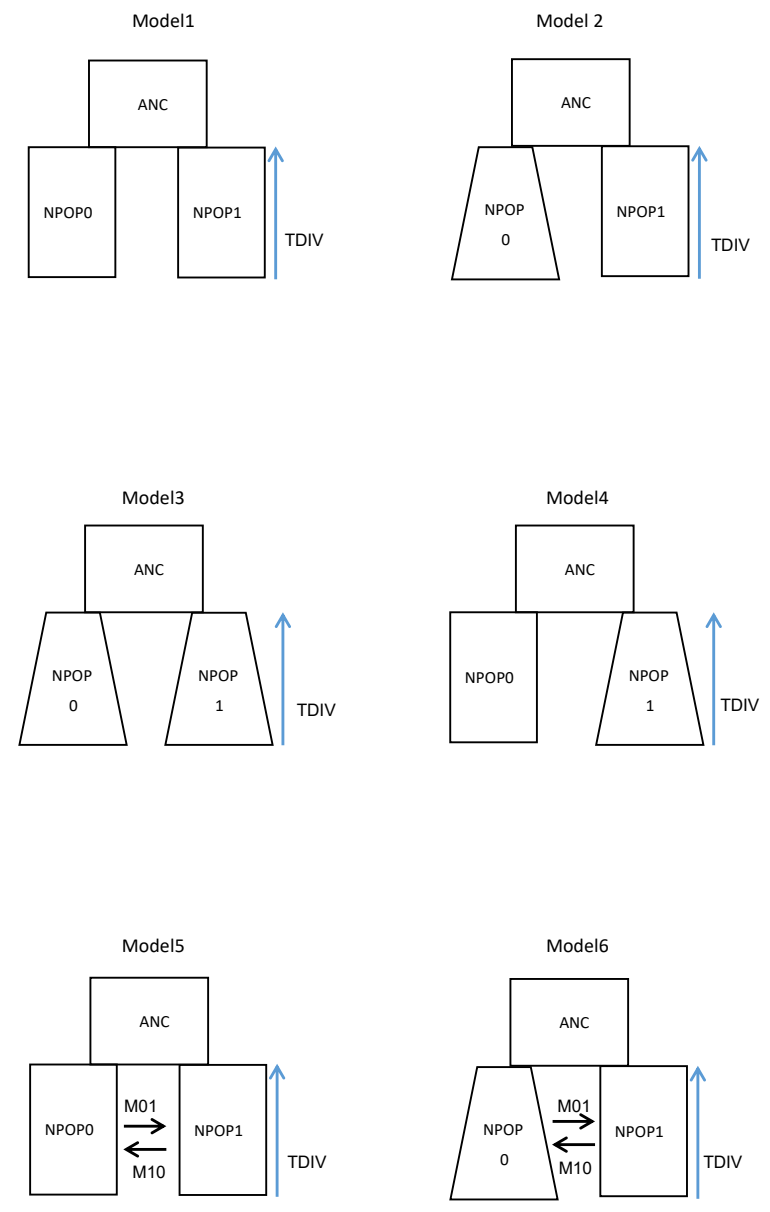
Table S1. Overview informatio of Illumina re-sequencing data per

\begin{tabular}{|c|c|c|c|c|c|}
\hline Sample ID & Location & Latitude & Longitude & $\begin{array}{l}\text { Mapping } \\
\text { rate (\%) }\end{array}$ & $\begin{array}{l}\text { Mean } \\
\text { Coverage } \\
\end{array}$ \\
\hline \multicolumn{6}{|l|}{ Palba } \\
\hline P.alba 1 & Aletai & 47.5669 & 87.2533 & $95.23 \%$ & 23.432 \\
\hline P.alba 2 & Aletai & 47.3483 & 87.8697 & $95.29 \%$ & 23.953 \\
\hline P.alba 4 & Aletai & 47.8562 & 86.5721 & $95.21 \%$ & 20.471 \\
\hline P.alba 5 & Aletai & 47.8330 & 86.6641 & $95.08 \%$ & 23.772 \\
\hline P.alba11 & Aletai & 47.6919 & 86.9401 & $94.02 \%$ & 25.479 \\
\hline P.alba 15 & Aletai & 47.6717 & 86.9699 & $94.60 \%$ & 21.449 \\
\hline P.alba 16 & Aletai & 47.6484 & 86.9746 & $94.54 \%$ & 27.622 \\
\hline P.alba 17 & Aletai & 47.6235 & 87.0252 & $93.87 \%$ & 24.601 \\
\hline P.alba 18 & Aletai & 47.6080 & 87.0711 & $94.63 \%$ & 25.185 \\
\hline P.alba 19 & Aletai & 47.5769 & 87.1332 & $94.49 \%$ & 23.165 \\
\hline P.alba 20 & Aletai & 47.5987 & 87.055 & $94.47 \%$ & 26.407 \\
\hline P.alba 21 & Aletai & 47.4538 & 87.5196 & $94.61 \%$ & 20.246 \\
\hline Palba 22 & Aletai & 47.4398 & 87.6599 & $94.18 \%$ & 26.869 \\
\hline P.alba24 & Aletai & 47.4210 & 87.7288 & $94.50 \%$ & 24.901 \\
\hline P.alba25 & Aletai & 47.3367 & 88.0025 & $94.09 \%$ & 23.821 \\
\hline P.alba 26 & Aletai & 47.4117 & 87.7174 & $93.96 \%$ & 21.650 \\
\hline P.alba 28 & Aletai & 47.5068 & 87.3218 & $94.09 \%$ & 18.222 \\
\hline P.alba 32 & Aletai & 47.3444 & 87.8892 & $94.43 \%$ & 20.958 \\
\hline P.alba33 & Aletai & 47.4911 & 87.3347 & $94.67 \%$ & 24.890 \\
\hline P.alba34 & Aletai & 47.3914 & 87.7817 & $94.20 \%$ & 20.117 \\
\hline P.alba $\mathrm{H}-1$ & Aletai & 47.9011 & 86.1904 & $94.14 \%$ & 23.788 \\
\hline P.albaH-2 & Aletai & 47.9073 & 86.0799 & $94.06 \%$ & 23.765 \\
\hline P.albaH-5 & Aletai & 47.9274 & 86.0087 & $94.07 \%$ & 26.171 \\
\hline P.albaH-7 & Aletai & 47.9938 & 85.6959 & $94.12 \%$ & 22.549 \\
\hline P.albaH-8 & Aletai & 48.0185 & 85.6155 & $93.90 \%$ & 26.838 \\
\hline P.albaH-9 & Aletai & 48.0324 & 85.6844 & $94.18 \%$ & 25.803 \\
\hline YBYhz & Aletai & 48.0216 & 85.6178 & $94.71 \%$ & 28.777 \\
\hline Mean & & & & $94.42 \%$ & 23.885 \\
\hline \multicolumn{6}{|c|}{ P. adenopoda } \\
\hline $\mathrm{AH} 2-1$ & Anhui & 30.2428 & 118.4423 & $95.17 \%$ & 22.644 \\
\hline AH3-1 & Anhui & 30.2435 & 118.50 & $95.55 \%$ & 25.427 \\
\hline AH4-1 & Anhui & 30.251 & 118.4645 & $95.62 \%$ & 33.695 \\
\hline AH5-1 & Anhui & 30.2449 & 118.5827 & $95.43 \%$ & 27.740 \\
\hline AH6-2 & Anhui & 30.2539 & 119.0318 & $94.90 \%$ & 23.745 \\
\hline AH7-2 & Anhui & 30.2718 & 118.5722 & $94.96 \%$ & 24.329 \\
\hline AH8-2 & Anhui & 30.2736 & 118.545 & $95.02 \%$ & 26.421 \\
\hline CQ2 & Chongqing & 29.0812 & 107.0663 & $95.09 \%$ & 16.917 \\
\hline
\end{tabular}


Table S2. Relative likelihood of the different models shown in Figure

S1

\begin{tabular}{|c|c|c|c|c|c|}
\hline model & $\operatorname{Max}\left(\log 10\left(\right.\right.$ Lhoodi $^{\mathrm{a}}$ & $\begin{array}{c}\text { No. Of } \\
\text { parameters(d) }\end{array}$ & $\mathrm{AICi}^{\mathrm{b}}$ & $\Delta \mathbf{i}^{\mathbf{b}}$ & $\begin{array}{c}\text { Model normalized } \\
\text { relative likelihood(wi)b }\end{array}$ \\
\hline Modell & -85025483.6 & 4 & 391556830.1 & 681380.8686 & $\sim 0$ \\
\hline Mode2 & -85103413.95 & 5 & 391915714.6 & 1040271.393 & $\sim 0$ \\
\hline Model3 & -85056421.02 & 6 & 391699306.2 & 823858.9528 & $\sim 0$ \\
\hline Model4 & -85033399.28 & 5 & 391593285.2 & 717843.9221 & $\sim 0$ \\
\hline Model5 & -85019567.11 & 6 & 391529587.7 & 654144.4252 & $\sim 0$ \\
\hline Model6 & -85048908.67 & 7 & 391664712.6 & 789263.3025 & $\sim 0$ \\
\hline Model7 & -84982673.75 & 8 & 391359691.5 & 484250.2237 & $\sim 0$ \\
\hline Model8 & -85023913.29 & 7 & 391549604.6 & 674153.3238 & $\sim 0$ \\
\hline Model10 & -85043369.01 & 6 & 391639199.5 & 763756.2255 & $\sim 0$ \\
\hline Model11 & -85022818.26 & 7 & 391544561.8 & 669112.5243 & $\sim 0$ \\
\hline Model12 & -85025552.15 & 6 & 391557149.8 & 681706.553 & $\sim 0$ \\
\hline Model13 & -85025440.78 & 7 & 391556638.9 & 681185.6752 & $\sim 0$ \\
\hline Model14 & -85061928.28 & 8 & 391724672.1 & 849230.8223 & $\sim 0$ \\
\hline Model15 & -84982165.92 & 9 & 391357354.8 & 481897.5801 & $\sim 0$ \\
\hline Model16 & -85040874.24 & 8 & 391627714.6 & 752265.385 & $\sim 0$ \\
\hline Model17 & -128561324.8 & 7 & 592046794 & 201171346.8 & $\sim 0$ \\
\hline Model18 & -84929383.02 & 8 & 391114278.6 & 238827.3427 & $\sim 0$ \\
\hline Model19 & -84974058.69 & 9 & 391320019.7 & 444566.4062 & $\sim 0$ \\
\hline Model20 & -84967887.1 & 8 & 391291596.4 & 416147.1839 & $\sim 0$ \\
\hline Model21 & -84962919.61 & 9 & 391268722.3 & 393273.0471 & $\sim 0$ \\
\hline Model22 & -84877520.15 & 9 & 390875443.3 & 0 & 1 \\
\hline Model23 & -84892761.84 & 12 & 390945639.8 & 70184.57637 & $\sim 0$ \\
\hline Model24 & -84900737.75 & 12 & 390982370.3 & 106912.9993 & $\sim 0$ \\
\hline Model25 & -84934178.07 & 12 & 391136368.6 & 260909.364 & $\sim 0$ \\
\hline
\end{tabular}

${ }^{\text {a }}$ Based on the best likelihood among the 50 independent runs for each model (Figure S1).

${ }^{\mathrm{b}}$ The calculation of $\mathrm{AICi}, \Delta \mathrm{i}$ and wi are according to the methods shown in Excoffier et al. (2013). 
Table S3. A comparison of the regions showing extreme genetic differentiation in P. alba and P. adenopoda with other genomic regions is presented (give the mean \pm standard deviation).

\begin{tabular}{|c|c|c|c|c|}
\hline Parameters & Species & $\begin{array}{l}\text { Regions displaying } \\
\text { high differentiation }\end{array}$ & $\begin{array}{l}\text { Regions displaying } \\
\text { low differentiation }\end{array}$ & Background \\
\hline \multirow[t]{2}{*}{$\theta \pi$} & P.alba & $0.0005( \pm 0.0003)^{* * *}$ & $0.0014( \pm 0.0007)^{* * *}$ & $0.0011( \pm 0.0006)$ \\
\hline & P.adenopoda & $0.0002( \pm 0.0002)^{* * *}$ & $0.0013( \pm 0.0007)^{* * *}$ & $0.0007( \pm 0.0005)$ \\
\hline \multirow[t]{2}{*}{ Tajima's D } & P.alba & $1.2889( \pm 0.5060)$ & $1.3644( \pm 0.6763)^{* * *}$ & $1.1893( \pm 0.6189)$ \\
\hline & P.adenopoda & $1.1161( \pm 0.6088)$ & $1.2098( \pm 0.8052)^{* *}$ & $1.0416( \pm 0.7827)$ \\
\hline \multirow[t]{2}{*}{ Fay \& Wu's H } & P.alba & $-0.5939( \pm 0.3420)^{*}$ & $-0.3731( \pm 0.3552)^{* * *}$ & $-0.5392( \pm 0.3493)$ \\
\hline & P.adenopoda & $-0.7136( \pm 0.4016)$ & $-0.4640( \pm 0.4417)^{* * *}$ & $-0.7501( \pm 0.4315)$ \\
\hline \multirow[t]{2}{*}{$r^{2}\left(F_{\mathrm{ST}}\right)$} & P.alba & $0.3323( \pm 0.2043)$ & $0.2568( \pm 0.1531)^{* * *}$ & $0.3289( \pm 0.1550)$ \\
\hline & P.adenopoda & $0.2354( \pm 0.2368)$ & $0.2634( \pm 0.1985)^{* *}$ & $0.3105( \pm 0.2376)$ \\
\hline \multirow[t]{2}{*}{$r^{2}$ (Dxy) } & P.alba & $0.2674( \pm 0.1470)^{* * *}$ & $0.4213( \pm 0.1776)^{* * *}$ & $0.3216( \pm 0.1523)$ \\
\hline & P.adenopoda & $0.2350( \pm 0.2104)^{* * *}$ & $0.4249( \pm 0.2706)^{* * *}$ & $0.3048( \pm 0.2341)$ \\
\hline \multirow[t]{2}{*}{ Fixed(\%) } & P.alba & $0.2146( \pm 0.0887)^{* * * *}$ & $0.0258( \pm 0.0389)^{* * *}$ & $0.0999( \pm 0.0851)$ \\
\hline & P.adenopoda & $0.2535( \pm 0.1122)^{* * *}$ & $0.0296( \pm 0.0497)^{* * *}$ & $0.1211( \pm 0.0904)$ \\
\hline Shared(\%) & & $0.0517( \pm 0.0680)^{*}$ & $0.1695( \pm 0.1009)^{* *}$ & $0.0908( \pm 0.0965)$ \\
\hline$F_{\mathrm{ST}}$ & & $0.8594( \pm 0.0396)^{* * *}$ & $0.2070( \pm 0.0496)^{* * *}$ & $0.5496( \pm 0.1194)$ \\
\hline Dxy & & $0.0280( \pm 0.0092)^{* * *}$ & $0.0212( \pm 0.0083)^{*}$ & $0.0220( \pm 0.0085)$ \\
\hline RND & & $1.2226( \pm 0.2831)^{* * *}$ & $0.9213( \pm 0.2040)^{* * *}$ & $1.0906( \pm 0.1958)$ \\
\hline
\end{tabular}

By Mann-Whitney U test, there were significant differences between the genome background and regions showing extreme genetic differentiation (* $P$-value $<0.05 ;{ }^{* *} P$-value $<1 \mathrm{e}-4 ;{ }^{* * *} P$-value $<2.2 \mathrm{e}-16$ ). 
Table S4. List of genes located in a region of significantly high genetic differentiation between $P$. alba and $P$. adenopoda.

\begin{tabular}{|c|c|c|c|}
\hline \multirow{2}{*}{\begin{tabular}{|l|} 
Poplar gene \\
Potri.001G009600
\end{tabular}} & \multicolumn{2}{|c|}{$\begin{array}{l}\text { Best ArabidopsiSynonyms } \\
\text { hit }\end{array}$} & \multirow{2}{*}{$\begin{array}{l}\text { Annotated des cription } \\
\text { chromomethylase } 3\end{array}$} \\
\hline & AT1G69770 & CMT3 & \\
\hline Potri.001G011500 & & & \\
\hline Potri.001G011900 & AT3G07160 & GSL10 & glucan synthase-like 10 \\
\hline Potri.001G012200 & AT5G13000 & GSL12 & glucan synthase-like 12 \\
\hline Potri.001G048800 & AT1G15520 & ABCG40 & pleiotropic drug resistance 12 \\
\hline Potri.001G049400 & AT2G32700 & LUH & LEUNIG_homolog \\
\hline \multicolumn{4}{|c|}{ Potri.001G053200 } \\
\hline Potri.001G065800 & AT5G13260 & & \\
\hline Potri.001G083400 & AT4G24140 & & alpha/beta-Hydrolases superfamily protein \\
\hline Potri.001G083900 & AT2G46660 & CYP78A6 & $\begin{array}{l}\text { cytochrome P } 450 \text {, family } 78 \text {, subfamily A, } \\
\text { polypeptide } 6\end{array}$ \\
\hline Potri.001G092500 & AT5G53160 & RCAR3 & regulatory components of $\mathrm{ABA}$ receptor 3 \\
\hline Potri.001G099400 & AT4G11280 & ACS6 & $\begin{array}{l}\text { 1-aminocyclopropane-1-carboxylic acid (acc) } \\
\text { synthase } 6\end{array}$ \\
\hline Potri.001G101300 & AT5G41360 & XPB2 & $\begin{array}{l}\text { homolog of Xeroderma pigmentosum } \\
\text { complementation group B } 2\end{array}$ \\
\hline Potri.001G117000 & AT1G12330 & & (1 of 1) PTHR31029:SF4 - F5O11.6 \\
\hline Potri.001G118500 & AT4G12310 & CYP706A5 & $\begin{array}{l}\text { cytochrome } \mathrm{P} 450 \text {, family } 706 \text {, subfamily A, } \\
\text { polypeptide } 5\end{array}$ \\
\hline Potri.001G119000 & AT1G62790 & & $\begin{array}{l}\text { Bifunctional inhibitor/lipid-transfer protein/seed } \\
\text { storage } 2 \mathrm{~S} \text { albumin superfamily protein }\end{array}$ \\
\hline \multicolumn{4}{|l|}{ Potri.001G125900 } \\
\hline \multicolumn{4}{|c|}{ Potri.001G190400 } \\
\hline Potri.001G196500 & AT1G17640 & & $\begin{array}{l}\text { RNA-binding (RRM/RBD/RNP motifs) family } \\
\text { protein }\end{array}$ \\
\hline Potri.001G198000 & AT1G54220 & & $\begin{array}{l}\text { Dihydrolipoamide acetyltransferase, long form } \\
\text { protein }\end{array}$ \\
\hline \multicolumn{4}{|l|}{ Potri.001G211900 } \\
\hline Potri.001G215700 & AT1G08420 & BSL2 & BRI1 suppressor 1 (BSU1)-like 2 \\
\hline Potri.001G233600 & AT5G59990 & & CCT motif family protein \\
\hline Potri.001G249900 & AT2G29660 & & zinc finger ( $\mathrm{C} 2 \mathrm{H} 2$ type) family protein \\
\hline
\end{tabular}


Table S5. List of genes located in a region of significantly low genetic differentiation between $P$. alba and $P$. adenopoda.

\begin{tabular}{|c|c|c|c|}
\hline Poplar gene & $\begin{array}{l}\text { Best Arabidopsis } \\
\text { hit }\end{array}$ & Synonyms & Annotated description \\
\hline \multicolumn{4}{|l|}{ Potri.001G031200 } \\
\hline \multicolumn{4}{|l|}{ Potri.001G035300 } \\
\hline Potri.001G037200 & AT3G14250 & & RING/U-box superfamily protein \\
\hline Potri.001G038000 & AT1G16190 & RAD23A & Rad23 UV excision repair protein family \\
\hline \multicolumn{4}{|l|}{ Potri.001G041800 } \\
\hline Potri.001G043900 & AT1G15990 & CNGC7 & cyclic nucleotide gated channel 7 \\
\hline Potri.001G044000 & AT1G73060 & LPA3 & Low PSII Accumulation 3 \\
\hline Potri.001G046100 & AT1G80820 & CCR2 & cinnamoyl coa reductase \\
\hline Potri.001G046400 & AT1G80820 & CCR2 & cinnamoyl coa reductase \\
\hline \multicolumn{4}{|c|}{ Potri.001G051800 } \\
\hline \multicolumn{4}{|c|}{ Potri.001G065300 } \\
\hline Potri.001G071500 & AT5G47900 & & Protein of unknown function (DUF1624) \\
\hline \multicolumn{4}{|l|}{ Potri.001G076300 } \\
\hline \multicolumn{4}{|l|}{ Potri.001G134500 } \\
\hline Potri.001G146800 & AT5G45540 & & Protein of unknown function (DUF594) \\
\hline Potri.001G172600 & AT1G52670 & & Single hybrid motif superfamily protein \\
\hline Potri.001G174500 & AT1G74810 & BOR5 & HCO3- transporter family \\
\hline Potri.001G181200 & AT1G79915 & & Putative methyltransferase family protein \\
\hline Potri.001G189500 & AT1G15210 & ABCG35 & pleiotropic drug resistance 7 \\
\hline Potri.001G208900 & AT5G27010 & & ARM repeat superfamily protein \\
\hline Potri.001G223900 & AT2G44490 & PEN2 & Glycosyl hydrolase superfamily protein \\
\hline Potri.001G236700 & AT3G46010 & ADF1 & actin depolymerizing factor 1 \\
\hline Potri.001G267700 & AT3G63530 & BB & RING/U-box superfamily protein \\
\hline \multicolumn{4}{|l|}{ Potri.001G268700 } \\
\hline \multicolumn{4}{|l|}{ Potri.001G270500 } \\
\hline \multicolumn{4}{|l|}{ Potri.001G283200 } \\
\hline \multicolumn{4}{|l|}{ Potri.001G333000 } \\
\hline Potri.001G334000 & AT3G01790 & & Ribosomal protein L13 family protein \\
\hline Potri.001G340200 & AT3G01680 & SEOR1 & $\begin{array}{l}\text { (1 of 3) PF14576 - Sieve element occlusion } \\
\text { N-terminus (SEO_N) }\end{array}$ \\
\hline Potri.001G340300 & AT3G01680 & SEOR1 & $\begin{array}{l}\text { (1 of 3) PF14576 - Sieve element occlusion } \\
\text { N-terminus (SEO_N) }\end{array}$ \\
\hline Potri.001G360800 & AT1G66980 & $\mathrm{SNC} 4$ & suppressor of npr 1-1 constitutive 4 \\
\hline Potri.001G386300 & AT1G53430 & & $\begin{array}{l}\text { Leucine-rich repeat transmembrane protein } \\
\text { kinase }\end{array}$ \\
\hline Potri.001G386700 & AT1G53450 & & \\
\hline
\end{tabular}


Table S6. Enriched gene ontology terms among CDR genes

\begin{tabular}{lcc}
\hline Gene ontology term & $P_{\mathrm{FDR}}$ value & Gene count \\
\hline response to lipid & 0.00790073 & $16(408)$ \\
single-organism developmental process & 0.01216598 & $33(1508)$ \\
cellular response to lipid & 0.01216598 & $10(201)$ \\
developmental process & 0.01216598 & $33(1543)$ \\
response to endogenous stimulus & 0.01216598 & $23(907)$ \\
response to oxygen-containing compound & 0.01216598 & $21(787)$ \\
response to hormone & 0.01216598 & $22(850)$ \\
brassinosteroid mediated signaling pathway & 0.01216598 & $4(25)$ \\
steroid hormone mediated signaling pathway & 0.01216598 & $4(25)$ \\
response to steroid hormone & 0.01216598 & $4(25)$ \\
cellular response to steroid hormone stimulus & 0.01216598 & $4(25)$ \\
response to alcohol & 0.01216598 & $13(364)$ \\
cellular response to oxygen-containing compoun & 0.01216598 & $12(318)$ \\
cellular response to brassinosteroid stimulus & 0.01216598 & $4(26)$ \\
response to acid chemical & 0.0122692 & $17(589)$ \\
anatomical structure development & 0.0122692 & $28(1281)$ \\
cellular response to hormone stimulus & 0.0160557 & $13(386)$ \\
cellular response to endogenous stimulus & 0.01650461 & $13(390)$ \\
single organism reproductive process & 0.01650461 & $20(795)$ \\
single-multicellular organism process & 0.01650461 & $28(1325)$ \\
multicellular organismal process & 0.01650461 & $30(1465)$ \\
cellular response to chemical stimulus & 0.01650461 & $16(563)$ \\
developmental process involved in reproduction & 0.01829557 & $18(694)$ \\
reproductive process & 0.01829557 & $21(881)$ \\
cellular response to organic substance & 0.01829557 & $14(463)$ \\
reproduction & 0.01829557 & $21(883)$ \\
gibberellic acid mediated signaling pathway & 0.01831615 & $4(34)$ \\
hormone-mediated signaling pathway & 0.01909502 & $12(362)$ \\
gibberellin mediated signaling pathway & 0.01909502 & $4(35)$ \\
cellular response to gibberellin stimulus & 0.02059513 & $4(36)$ \\
reproductive structure development & 0.02274415 & $16(600)$ \\
reproductive system development & 0.02274415 & $16(600)$ \\
flower development & 0.02502946 & $9(228)$ \\
response to organic substance & 0.02502946 & $23(1056)$ \\
& & \\
\end{tabular}


Table S7. List of the genes related to reproduction and adaptation located in regions displaying significantly high genetic differentiation between $P$. alba and $P$. adenopoda.

\begin{tabular}{|c|c|c|c|}
\hline Poplar gene & $\begin{array}{l}\text { Best } \\
\text { Arabidopsis hit }\end{array}$ & Synonyms & Annotated description \\
\hline Potri.004G133500 & AT3G48750 & $\mathrm{CDC} 2$ & cell division control 2 \\
\hline Potri.008G206000 & AT1G48270 & GCR1 & G-protein-coupled receptor 1 \\
\hline Potri.008G217300 & AT4G02570 & CUL1 & cullin 1 \\
\hline Potri.010G250800 & AT3G21180 & NFXL2 & $\begin{array}{l}\text { sequence-specific DNA binding } \\
\text { transcription factors; zinc ion } \\
\text { binding; sequence-specific DNA } \\
\text { binding transcription factors } \\
\text { autoinhibited } \mathrm{Ca}(2+) \text {-ATPase } 9\end{array}$ \\
\hline Potri.012G095800 & AT5G63420 & $\mathrm{emb} 2746$ & $\begin{array}{l}\text { RNA-metabolising } \\
\text { metallo-beta-lactamase family } \\
\text { protein }\end{array}$ \\
\hline Potri.012G132400 & AT4G25420 & GA20OX1 & $\begin{array}{l}\text { 2-oxoglutarate }(2 \mathrm{OG}) \text { and } \\
\text { Fe(II)-dependent oxygenase } \\
\text { superfamily protein }\end{array}$ \\
\hline Potri.014G066700 & AT2G45190 & AFO & $\begin{array}{l}\text { Plant-specific transcription } \\
\text { factor YABBY family protein }\end{array}$ \\
\hline Potri.014G164700 & AT5G35750 & HK2 & histidine kinase 2 \\
\hline Potri.015G043200 & AT1G17980 & PAPS1 & poly(A) polymerase 1 \\
\hline Potri.019G044400 & AT5G17690 & TFL2 & $\begin{array}{l}\text { like heterochromatin protein } \\
\text { (LHP1) }\end{array}$ \\
\hline Potri.001G009600 & AT1G69770 & CMT3 & chromomethylase 3 \\
\hline Potri.002G161700 & AT4G00310 & EDA8 & Putative membrane lipoprotein \\
\hline Potri.012G100400 & AT5G63510 & MLE2.14 & $\begin{array}{l}\text { gamma carbonic anhydrase like } \\
1\end{array}$ \\
\hline Potri.013G086600 & AT5G16490 & RIC4 & $\begin{array}{l}\text { ROP-interactive CRIB } \\
\text { motif-containing protein } 4\end{array}$ \\
\hline Potri.001G092500 & AT5G53160 & RCAR3 & $\begin{array}{l}\text { regulatory components of } \mathrm{ABA} \\
\text { receptor } 3\end{array}$ \\
\hline Potri.001G215700 & AT1G08420 & BSL2 & $\begin{array}{l}\text { BRI1 suppressor } 1 \text { (BSU1)-like } \\
2\end{array}$ \\
\hline Potri.002G206100 & AT2G47770 & TSPO & $\begin{array}{l}\text { TSPO(outer membrane } \\
\text { tryptophan-rich sensory } \\
\text { protein)-related }\end{array}$ \\
\hline Potri.002G213300 & AT4G03080 & BSL1 & $\begin{array}{l}\text { BRI1 suppressor } 1 \text { (BSU1)-like } \\
1\end{array}$ \\
\hline
\end{tabular}


Table S8. Functional gene categories enriched for genes located within regions dis significantly high genetic differentiation between $P$. alba and P.adenopoda.

\begin{tabular}{|c|c|c|c|c|}
\hline GO Term & Ontology & Description & $P$-value & FDR \\
\hline GO:0044702 & $\mathrm{P}$ & single organism reproductive process & 4.90E-07 & $1.60 \mathrm{E}-03$ \\
\hline GO:0044767 & $P$ & single-organism developmental process & $6.50 \mathrm{E}-07$ & $1.60 \mathrm{E}-03$ \\
\hline GO:0032502 & $\mathrm{P}$ & developmental process & $1.10 \mathrm{E}-06$ & $1.60 \mathrm{E}-03$ \\
\hline GO:0048856 & $P$ & anatomical structure development & $1.20 \mathrm{E}-06$ & $1.60 \mathrm{E}-03$ \\
\hline GO:0009908 & $\mathrm{P}$ & flower development & $1.60 \mathrm{E}-06$ & $1.70 \mathrm{E}-03$ \\
\hline GO:0090567 & $P$ & reproductive shoot system development & 2.20E-06 & $1.83 \mathrm{E}-03$ \\
\hline GO:0003006 & $\mathrm{P}$ & developmental process involved in reproduction & 2.40E-06 & $1.83 \mathrm{E}-03$ \\
\hline GO:0033993 & $P$ & response to lipid & 3.00E-06 & $2.00 \mathrm{E}-03$ \\
\hline GO:0009719 & $\mathrm{P}$ & response to endogenous stimulus & $3.60 \mathrm{E}-06$ & $2.13 \mathrm{E}-03$ \\
\hline GO:0022414 & $\mathrm{P}$ & reproductive process & $6.20 \mathrm{E}-06$ & $3.11 \mathrm{E}-03$ \\
\hline GO:0048608 & $\mathrm{P}$ & reproductive structure development & 7.00E-06 & $3.11 \mathrm{E}-03$ \\
\hline GO:0061458 & $P$ & reproductive system development & 7.00E-06 & $3.11 \mathrm{E}-03$ \\
\hline GO:0009725 & $P$ & response to hormone & $9.90 \mathrm{E}-06$ & $3.80 \mathrm{E}-03$ \\
\hline GO:0044707 & $\mathrm{P}$ & single-multicellular organism process & $1.00 \mathrm{E}-05$ & $3.80 \mathrm{E}-03$ \\
\hline GO:1901700 & $P$ & response to oxygen-containing compound & $1.10 \mathrm{E}-05$ & $3.91 \mathrm{E}-03$ \\
\hline GO:0009909 & $\mathrm{P}$ & regulation of flower development & $1.30 \mathrm{E}-05$ & 4.33E-03 \\
\hline GO:0032501 & $P$ & multicellular organismal process & $1.50 \mathrm{E}-05$ & 4.70E-03 \\
\hline GO:0007275 & $\mathrm{P}$ & multicellular organismal development & $3.30 \mathrm{E}-05$ & $9.76 \mathrm{E}-03$ \\
\hline GO:0001101 & $P$ & response to acid chemical & 3.60E-05 & $1.01 \mathrm{E}-02$ \\
\hline GO:0048731 & $\mathrm{P}$ & system development & 4.00E-05 & $1.07 \mathrm{E}-02$ \\
\hline GO:0097305 & $P$ & response to alcohol & 4.90E-05 & $1.24 \mathrm{E}-02$ \\
\hline GO:0010033 & $P$ & response to organic substance & $5.90 \mathrm{E}-05$ & $1.43 \mathrm{E}-02$ \\
\hline GO:2000241 & $P$ & regulation of reproductive process & $6.30 \mathrm{E}-05$ & $1.46 \mathrm{E}-02$ \\
\hline GO:0048831 & $P$ & regulation of shoot system development & $8.50 \mathrm{E}-05$ & $1.85 \mathrm{E}-02$ \\
\hline GO:0048367 & $P$ & shoot system development & $8.70 \mathrm{E}-05$ & $1.85 \mathrm{E}-02$ \\
\hline GO:0009791 & $P$ & post-embryonic development & 0.00011 & $2.25 \mathrm{E}-02$ \\
\hline GO:0071396 & $P$ & cellular response to lipid & 0.00015 & $2.96 \mathrm{E}-02$ \\
\hline GO:0009742 & $P$ & brassinosteroid mediated signaling pathway & 2.00E-04 & $3.55 \mathrm{E}-02$ \\
\hline GO:0043401 & $P$ & steroid hormone mediated signaling pathway & 2.00E-04 & 3.55E-02 \\
\hline GO:0071383 & $P$ & cellular response to steroid hormone stimulus & 2.00E-04 & 3.55E-02 \\
\hline GO:0071367 & $P$ & cellular response to brassinosteroid stimulus & 0.00022 & $3.78 \mathrm{E}-02$ \\
\hline GO:0048545 & $P$ & response to steroid hormone & 0.00025 & 4.16E-02 \\
\hline GO:0009628 & $P$ & response to abiotic stimulus & 0.00029 & 4.68E-02 \\
\hline GO:0032870 & $\mathrm{P}$ & cellular response to hormone stimulus & 4.00E-04 & $6.27 \mathrm{E}-02$ \\
\hline GO:0071495 & $P$ & cellular response to endogenous stimulus & 0.00043 & $6.54 \mathrm{E}-02$ \\
\hline GO:1901701 & $\mathrm{P}$ & cellular response to oxygen-containing compound & $5.00 \mathrm{E}-04$ & $7.34 \mathrm{E}-02$ \\
\hline
\end{tabular}


Table S9.

Gene introduction

1. Cell division. The CDC2 (cyclin-dependent kinase) gene encodes a catalytic subunit of a cyclin-dependent kinase and is involved in many cellular processes, including the control of cell division. Many of the cdc2 gene and its related genes encode proteins that interact with cyclins; these genes are collectively referred to as cyclin-dependent kinases (Rossmacdonald, Graeser, Kappes, Franklin, \& Williamson, 1994). Fobert et al. isolated four cdc2-related genes from Antirrhinum (Fobert, Gaudin, Lunness, Coen, \& Doonan, 1996). Genetic studies of cell division in yeast have identified the product of the cdc2 gene as a key component of these cascades. And the histological distribution of their transcripts suggests that members of the Antirrhinum cdc2 gene family are differentially expressed during the cell division cycle (Fobert et al., 1996).

2. Pollen rejection pathways. Mutations in CUL1 (Cullin1) establish unilateral incompatibility with self-incompatibility (SI) populations and strengthen reproductive isolation (Markova et al., 2016). Li et al. used a self-incompatible wild tomato species Solanum Arcanum, silence CUL1 expression in pollen by RNA interference (RNAi), resulting in pollen rejection in normally compatible sibling hybridization, while the same pollen remained fully compatible on sc logins expressing inactive s-RNase. The results strongly suggest that CUL1 has a role in protecting pollen from s-rnases in SI and UI (interspecific incompatibility) and provides further evidence for overlap between intraspecific and interspecific pollen rejection pathways (Li \& Chetelat, 2014).

3. Embryogenesis. Emb2746 genes play an important role in fruit development. In the GT-1 subfamily, EMB2746 (At5g63420), which exists in Arabidopsis thaliana, is 